\title{
A Lorentzian Stochastic Estimation for a Robust Iterative Multiframe Super-Resolution Reconstruction with Lorentzian-Tikhonov Regularization
}

\author{
V. Patanavijit and S. Jitapunkul \\ Department of Electrical Engineering, Faculty of Engineering, Chulalongkorn University, Bangkok 10330, Thailand
}

Received 31 August 2006; Revised 12 March 2007; Accepted 16 April 2007

Recommended by Richard R. Schultz

Recently, there has been a great deal of work developing super-resolution reconstruction (SRR) algorithms. While many such algorithms have been proposed, the almost SRR estimations are based on L1 or L2 statistical norm estimation, therefore these SRR algorithms are usually very sensitive to their assumed noise model that limits their utility. The real noise models that corrupt the measure sequence are unknown; consequently, SRR algorithm using L1 or L2 norm may degrade the image sequence rather than enhance it. Therefore, the robust norm applicable to several noise and data models is desired in SRR algorithms. This paper first comprehensively reviews the SRR algorithms in this last decade and addresses their shortcomings, and latter proposes a novel robust SRR algorithm that can be applied on several noise models. The proposed SRR algorithm is based on the stochastic regularization technique of Bayesian MAP estimation by minimizing a cost function. For removing outliers in the data, the Lorentzian error norm is used for measuring the difference between the projected estimate of the high-resolution image and each low-resolution image. Moreover, Tikhonov regularization and Lorentzian-Tikhonov regularization are used to remove artifacts from the final answer and improve the rate of convergence. The experimental results confirm the effectiveness of our method and demonstrate its superiority to other super-resolution methods based on L1 and L2 norms for several noise models such as noiseless, additive white Gaussian noise (AWGN), poisson noise, salt and pepper noise, and speckle noise.

Copyright (c) 2007 V. Patanavijit and S. Jitapunkul. This is an open access article distributed under the Creative Commons Attribution License, which permits unrestricted use, distribution, and reproduction in any medium, provided the original work is properly cited.

\section{GENERAL INTRODUCTION}

Traditionally, theoretical and practical limitations constrain the achievable resolution of any devices. super-resolution reconstruction (SRR) algorithms investigate the relative motion information between multiple low-resolution (LR) images (or a video sequence) and increase the spatial resolution by fusing them into a single frame. In doing so, SRR also removes the effect of possible blurring and noise in the LR images [1-8]. Recent work relates this problem to restoration theory $[4,9]$. As such, the problem is shown to be an inverse problem, where an unknown image is to be reconstructed, based on measurements related to it through linear operators and additive noise. This linear relation is composed of geometric warp, blur, and decimation operations. The SRR problem is modelled by using sparse matrices and analyzed from many reconstruction methods [5] such as the nonuniform interpolation, frequency domain, maximum likelihood
(ML), maximum a posteriori (MAP), and projection onto convex sets (POCS). The general introduction of SRR algorithms in the last decade is reviewed in Section 1.1 and the SRR algorithm in estimation point of view is comprehensively reviewed in Section 1.2.

\subsection{Introduction of SRR}

The super-resolution restoration idea was first presented by Huang and Tsan [10] in 1984. They used the frequency domain approach to demonstrate the ability to reconstruct one improved resolution image from several downsampled noise-free versions of it, based on the spatial aliasing effect. Next, a frequency domain recursive algorithm for the restoration of super-resolution images from noisy and blurred measurements is proposed by Kim et al. [11] in 1990. The algorithm using a weighted recursive leastsquares algorithm is based on sequential estimation theory in the frequency-wavenumber domain, to achieve simultaneous 
improvement in signal-to-noise ratio and resolution from available registered sequence of low-resolution noisy frames. In 1993, Kim and Su [12] also incorporated explicitly the deblurring computation into the high-resolution image reconstruction process because separate deblurring of input frames would introduce the undesirable phase and high wavenumber distortions in the DFT of those frames. Subsequently, Ng and Bose [13] proposed the analysis of the displacement errors on the convergence rate to the iterative approach for solving the transform-based preconditioned system of equation in 2002, hence it is established that the use of the MAP, L2 norm or $\mathrm{H} 1$ norm regularization functional leads to a proof of linear convergence of the conjugate gradient method in terms of the displacement errors caused by the imperfect subpixel locations. Later, Bose et al. [14] proposed the fast SRR algorithm, using MAP with MRF for blurred observation in 2006. This algorithm uses the reconditioned conjugated gradient method and FFT. Although the frequency domain methods are intuitively simple and computationally cheap, the observation model is restricted to only global translational motion and LSI blur. Due to the lack of data correlation in the frequency domain, it is also difficult to apply the spatial domain a priori knowledge for regularization.

The POCS formulation of the SRR was first suggested by Stark and Oskoui [8] in 1987. Their method was extended by Tekalp [8] to include observation noise in 1992. Although the advantage of POCS is that it is simple and can utilize a convenient inclusion of a priori information, these methods have the disadvantages of nonuniqueness of solution, slow convergence, and a high computational cost. Next, Patti and Altunbasak [15] proposed an SRR using ML estimator with POCSbased regularization in 2001 and Altunbasak et al. [16] proposed a super-resolution restoration for the MPEG sequences in 2002. They proposed a motion-compensated, transform-domain super-resolution procedure that directly incorporates the transform-domain quantization information by working with the compressed bit stream. Later, Gunturk et al. [17] proposed an ML super-resolution with regularization based on compression quantization, additive noise and image prior information in 2004. Next, Hasegawa et al. proposed iterative SSR using the adaptive projected subgradient method for MPEG sequences in 2005 [18].

The MRF or Markov/Gibbs random fields [19-26] are proposed and developed for modeling image texture during 1990-1994. Due to markov random field (MRF) that can model the image characteristic especially on image texture, Bouman and Sauer [27] proposed the single image restoration algorithm using MAP estimator with the generalized Gaussian-Markov random field (GGMRF) prior in 1993. Schultz and Stevenson [28] proposed the single image restoration algorithm using MAP estimator with the Huber-Markov random field (HMRF) prior in 1994. Next, the super-resolution restoration algorithm using MAP estimator (or the Regularized ML estimator), with the HMRF prior was proposed by Schultz and Stevenson [29] in 1996. The blur of the measured images is assumed to be simple averaging and the measurements additive noise is assumed to be independent and identically distributed (i.i.d.) Gaussian vector. In 2006, Pan and Reeves [30] proposed single image MAP estimator restoration algorithm with the efficient HMRF prior using decomposition-enabled edge-preserving image restoration in order to reduce the computational demand.

Typically, the regularized ML estimation (or MAP) [2, $4,9,31]$ is used in image restoration, therefore the determination of the regularization parameter is an important issue in the image restoration. Thompson et al. [32] proposed the methods of choosing the smoothing parameter in image restoration by regularized ML in 1991. Next, Mesarovic et al. [33] proposed the single image restoration using regularized ML for unknown linear space-invariant (LSI) point spread function (PSF) in 1995. Subsequently, Geman and Yang [34] proposed single image restoration using regularized $\mathrm{ML}$ with robust nonlinear regularization in 1995. This approach can be done efficiently by Monte Carlo Methods, for example, by FFT-based annealing using Markov chain that alternates between (global) transitions from one array to the other. Latter, Kang and Katsaggelos proposed the use of a single image regularization functional [35], which is defined in terms of restored image at each iteration step, instead of a constant regularization parameter, in 1995 and proposed regularized ML for SRR [36], in which no prior knowledge of the noise variance at each frame or the degree of smoothness of the original image is required, in 1997. In 1999, Molina et al. [37] proposed the application of the hierarchical ML with Laplacian regularization to the single image restoration problem and derived expressions for the iterative evaluation of the two hyperparameters (regularized parameters) applying the evidence and maximum a posteriori (MAP) analysis within the hierarchical regularized ML paradigm. In 2003, Molina et al. [38] proposed the mutiframe super-resolution reconstruction using ML with Laplacian regularization. The regularized parameter is defined in terms of restored image at each iteration step. Next, Rajan and Chaudhuri [39] proposed superresolution approach, based on ML with MRF regularization, to simultaneously estimate the depth map and the focused image of a scene, both at a super-resolution from its defocused observed images in 2003. Subsequently, He and Kondi $[40,41]$ proposed image resolution enhancement with adaptively weighted low-resolution images (channels) and simultaneous estimation of the regularization parameter in 2004 and proposed a generalized framework [42] of regularized image/video iterative blind deconvolution/superresolution (IBD-SR) algorithm using some information from the more matured blind deconvolution techniques form image restoration in 2005. Latter, they [43] proposed SRR algorithm that takes into account inaccurate estimates of the registration parameters and the point spread function in 2006. In 2006, Vega et al. [44] proposed the problem of deconvolving color images observed with a single coupled charged device (CCD) from the super-resolution point of view. Utilizing the regularized ML paradigm, an estimate of the reconstructed image and the model parameters is generated. 
Elad and Feuer [45] proposed the hybrid method combining the ML and nonellipsoid constraints for the superresolution restoration in 1997, and the adaptive filtering approach for the super-resolution restoration in 1999 [46, 47]. Next, they proposed two iterative algorithms, the R-SD and the R-LMS [48], to generate the desired image sequence at the practically computational complexity. These algorithms assume the knowledge of the blur, the down-sampling, the sequences motion, and the measurements noise characteristics, and apply a sequential reconstruction process. Subsequently, the special case of super-resolution restoration (where the warps are pure translations, the blur is space invariant and the same for all the images, and the noise is white) is proposed for a fast super-resolution restoration in 2001 [49]. Later, Nguyen et al. [50] proposed fast SRR algorithm using regularized ML by using efficient block circulant preconditioners and the conjugate gradient method in 2001. In 2002, Elad [51] proposed the bilateral filter theory and showed how the bilateral filter can be improved and extended to treat more general reconstruction problems. Consequently, the alternate super-resolution approach, L1 Norm estimator and robust regularization based on a bilateral total variance (BTV), was presented by Farsiu et al. $[52,53]$ in 2004. This approach performance is superior to what was proposed earlier in $[45,46,48]$ and this approach has fast convergence but this SRR algorithm effectively applies only on AWGN models. Next, they proposed a fast SRR of color images [54] using ML estimator with BTV regularization for luminance component and Tikhonov regularization for chrominance component in 2006. Subsequently, they proposed the dynamic super-resolution problem of reconstructing a high-quality set of monochromatic or color super-resolved images from low-quality monochromatic, color, or mosaiced frames [55]. This approach includes a joint method for simultaneous SR, deblurring, and demosaicing, this way taking into account practical color measurements encountered in video sequences. Later, we [56] proposed the SRR using a regularized ML estimator with affine block-based registration for the real image sequence. Moreover, Rochefort et al. [57] proposed super-resolution approach based on regularized ML [51] for the extended original observation model devoted to the case of nonisometirc interframe motion such as affine motion in 2006.

Baker and Kanade [58] proposed another superresolution algorithm (hallucination or recognition-based super-resolution) in 2002 that attempts to recognize local features in the low-resolution image and then enhances their resolution in an appropriate manner. Due to the training data-base, this algorithm performance depends on the image type (such as face or character) and this algorithm is not robust enough to be sued in typical surveillance video. Sun et al. [59] proposed hallucination super-resolution (for single image) using regularization ML with primal sketches as the basic recognition elements in 2003.

During 2004-2006, Vandewalle et al. [60-63] have proposed a fast super-resolution reconstruction based on a nonuniform interpolation using a frequency domain registration. This method has low computation and can be used in the real-time system but the degradation models are limited therefore this algorithm can apply on few applications. In 2006, Trimeche et al. [64] proposed SRR algorithm using an integrated adaptive filtering method to reject the outlier image regions for which registration has failed.

\subsection{Introduction of SRR estimation technique in super-resolution reconstruction}

This section reviews the literature from the estimation point of view because the SRR estimation is one of the most crucial parts of the SRR research areas and directly affects the SRR performance.

Bouman and Sauer [27] proposed the single image restoration algorithm using ML estimator (L2 Norm) with the GGMRF regularization in 1993. Schultz and Stevenson [28] proposed the single image restoration algorithm using ML estimator (L2 Norm) with the HMRF regularization in 1994 and proposed the SRR algorithm [29] using ML estimator (L2 Norm) with the HMRF regularization in 1996. The blur of the measured images is assumed to be simple averaging and the measurements additive noise is assumed to be independent and identically distributed (i.i.d.) Gaussian vector. Elad and Feuer [45] proposed the hybrid method combining the ML estimator (L2 Norm) and nonellipsoid constraints for the super-resolution restoration in 1997 [46, 47]. Next, they proposed two iterative algorithms, the R-SD and the R-LMS (L2 Norm) [48], to generate the desired image sequence at the practically computational complexity in 1999. These algorithms assume the knowledge of the blur, the downsampling, the sequences motion, and the measurements noise characteristics, and apply a sequential reconstruction process. Subsequently, the special case of super-resolution restoration (where the warps are pure translations, the blur is space invariant and the same for all the images, and the noise is white) is proposed for a fast super-resolution restoration using ML estimator (L2 Norm) in 2001 [49]. Later, Nguyen et al. [50] proposed fast SRR algorithm using regularized ML (L2 Norm) by using efficient block circulant preconditioners and the conjugate gradient method in 2001. In 2002, Patti and Altunbasak [15] proposed an SRR algorithm using ML (L2 Norm) estimator with POCS-based regularization. Altunbasak et al. [16] proposed an SRR algorithm using ML (L2 Norm) estimator for the MPEG sequences in 2002. Rajan and Chaudhuri [39] proposed SRR using ML (L2 Norm) with MRF regularization to simultaneously estimate the depth map and the focused image of a scene in 2003. The alternate superresolution approach, ML estimator (L1 Norm), and robust regularization based on a bilateral total variance (BTV), were presented by Farsiu et al. $[52,53]$ in 2004 . Next, they proposed a fast SRR of color images [54] using ML estimator (L1 Norm) with BTV regularization for luminance component and Tikhonov regularization for chrominance component in 2006. Subsequently, they proposed the dynamic super-resolution problem of reconstructing a high-quality set of monochromatic or color super-resolved images from low-quality monochromatic, color, or mosaiced frames [55]. 
This approach includes a joint method for simultaneous SR, deblurring, and Demosaicing, this way taking into account practical color measurements encountered in video sequences. Later, we [56] proposed the SRR using a regularized ML estimator (L2 Norm) with affine block-based registration for the real image sequence. Moreover, Rochefort et al. [57] proposed super-resolution approach based on regularized ML (L2 Norm) [51] for the extended original observation model devoted to the case of nonisometirc interframe motion such as affine motion in 2006. In 2006, Pan and Reeves [30] proposed single image restoration algorithm using ML estimator (L2 Norm) with the efficient HMRF regularization and using decomposition-enabled edge-preserving image restoration in order to reduce the computational demand.

The success of SRR algorithm is highly dependent on the accuracy of the model of the imaging process. Unfortunately, these models are not supposed to be exactly true, as they are merely mathematically convenient formulations of some general prior information. When the data or noise model assumptions do not faithfully describe the measure data, the estimator performance degrades. Furthermore, existence of outliers defined as data points with different distributional characteristics than the assumed model will produce erroneous estimates. Almost noise models used in SRR algorithms are based on additive white Gaussian noise model, therefore SRR algorithms can effectively apply only on the image sequence that is corrupted by AWGN. Due to this noise model, L1 norm or L2 norm errors are effectively used in SRR algorithm. Unfortunately, the real noise models that corrupt the measure sequence are unknown, therefore SRR algorithm using L1 norm or L2 norm may degrade the image sequence rather than enhance it. Therefore, the robust norm error is desired for using in SRR algorithm that can apply on several noise models. For normally distributed data, the L1 norm produces estimates with higher variance than the optimal L2 (quadratic) norm, but the L2 norm is very sensitive to outliers because the influence function increases linearly and without bound. From the robust statistical estimation [6568], Lorentzian norm is designed to be more robust than L1 and L2. Whereas Lorentzian norm is designed to reject outliers, the norm must be more forgiving about outliers; that is, it should increase less rapidly than L2.

This paper describes a novel super-resolution reconstruction (SRR) algorithm which is robust to outliers caused by several noise models, therefore the proposed SRR algorithm can apply on the real image sequence that is corrupted by unknown real noise models. For the data fidelity cost function, the Lorentzian error norm [65-68] is used for measuring the difference between the projected estimate of the highresolution image and each low-resolution image. Moreover, Tikhonov regularization and Lorentzian-Tikhonov regularization are used to remove artifacts from the final answer and improve the rate of convergence. We demonstrate that our method's performance is superior to what was proposed earlier in $[3,15,28,29,39,45-49,52-56,69]$, and so forth.

The organization of this paper is as follows. Section 2 reviews explain the main concepts of robust estimation tech- nique in SRR framework. Section 3 introduces the proposed super-resolution reconstruction using L1 with Tikhonov regularization, L2 with Tikhonov regularization, Lorentzian norm with Tikhonov regularization and Lorentzian norm with Lorentzian-Tikhonov regularization. Section 4 outlines the proposed solution and presents the comparative experimental results obtained by using the proposed Lorentzian norm method and by using the L1 and L2 norm methods. Finally, Section 5 provides the summary and conclusion.

\section{INTRODUCTION OF ROBUST ESTIMATION FOR SRR FRAMEWORK}

The first step to reconstruct the super-resolution (SR) image is to formulate an observation model that relates the original HR image to the observed LR sequences. We present the observation model for the general super-resolution reconstruction from image sequences. Based on the observation model, probabilistic super-resolution restoration formulations and solutions such as ML estimators provide a simple and effective way to incorporate various regularizing constraints. Regularization reduces the visibility of artifacts created during the inversion process. Then, we rewrite the definition of these ML estimators in the super-resolution context as the following minimization problem.

\subsection{Observation model}

In this section, we propose the problem and the model of super-resolution reconstruction. Define that a lowresolution image sequence is $\left\{\underline{\mathbf{Y}}_{k}\right\}, N_{1} \times N_{2}$ pixels, as our measured data. An HR image $\underline{\mathbf{X}}, q N_{1} \times q N_{2}$ pixels, is to be estimated from the LR sequences, where $q$ is an integer-valued interpolation factor in both the horizontal and vertical directions. To reduce the computational complexity, each frame is separated into overlapping blocks (the shadow blocks as shown in Figures 1(a) and 1(b)).

For convenience of notation, all overlapping blocked frames will be presented as vector, ordered column-wise lexicographically. Namely, the overlapping blocked LR frame is $\underline{Y}_{k} \in \mathbb{R}^{M^{2}}\left(M^{2} \times 1\right)$ and the overlapping blocked HR frame is $\underline{X} \in \mathbb{R}^{q^{2} M^{2}}\left(L^{2} \times 1\right.$ or $\left.q^{2} M^{2} \times 1\right)$. We assume that the two images are related via the following equation:

$$
\underline{Y}_{k}=D_{k} H_{k} F_{k} \underline{X}+\underline{V}_{k}, \quad k=1,2, \ldots, N,
$$

where $\underline{X}$ is blurred, decimated, down sampled, and contaminated by additive noise, giving $\underline{Y}_{k}(t)$. The matrix $F_{k}(F \in$ $\mathbb{R}^{q^{2} M^{2} \times q^{2} M^{2}}$ ) stands for the geometric warp (translation) between the images $\underline{X}$ and $\underline{Y}_{k} . H_{k}$ is the blur matrix which is a space and time invariant and $H_{k} \in \mathbb{R}^{q^{2} M^{2} \times q^{2} M^{2}} . D_{k}$ is the decimation matrix assumed constant and $D_{k} \in \mathbb{R}^{M^{2} \times q^{2} M^{2}} \cdot \underline{V}_{k}$ is a system noise and $\underline{V}_{k} \in \mathbb{R}^{M^{2}}$.

Typically, many available estimators that estimate an HR image from a set of noisy LR images are not exclusively based on the LR measurement. They are also based on many assumptions such as noise or motion models and these models are not supposed to be exactly true, as they are merely mathematically convenient formulations of some general prior information. When the fundamental assumptions of data and 


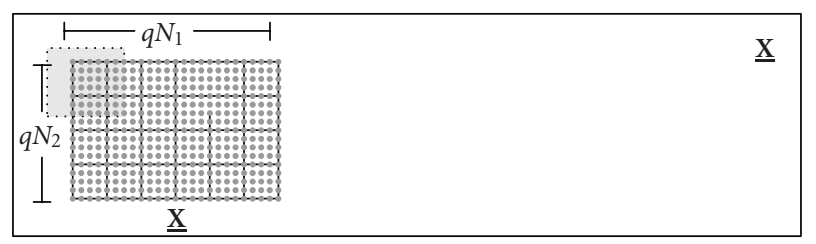

(a) High-resolution image

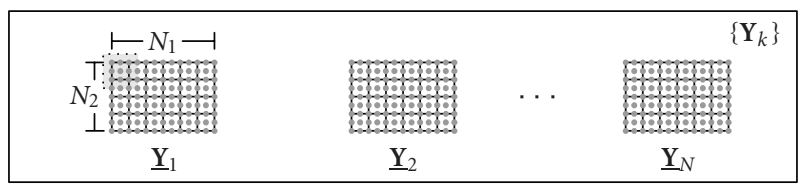

(b) Low-resolution image sequence

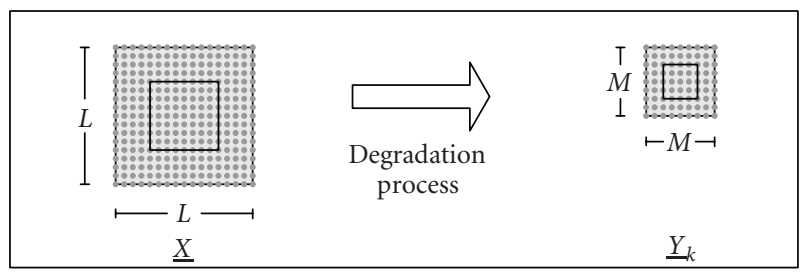

(c) The relation between overlapping blocked HR image and overlapping blocked LR image sequence

Figure 1: The observation model.

noise models do not faithfully describe the measured data, the estimator performance degrades. Moreover, existence of outliers defined as data points with different distributional characteristics than the assumed model will produce erroneous estimates. Estimators promising optimality for a limited class of data and noise models may not be the most effective overall approach. Often, suboptimal estimation methods that are not as sensitive to modeling and data errors may produce better and more stable results (robustness).

A popular family of estimators is the ML-type estimators ( $\mathrm{M}$ estimators) [50]. We rewrite the definition of these estimators in the super-resolution reconstruction framework as the following minimization problem:

$$
\underline{\hat{X}}=\underset{\underline{X}}{\operatorname{ArgMin}}\left\{\sum_{k=1}^{N} \rho\left(D_{k} H_{k} F_{k} \underline{X}-\underline{Y}_{k}\right)\right\}
$$

where $\rho(\cdot)$ is a robust error norm. To minimize (2), the intensity at each pixel of the expected image must be close to those of original image.

\section{2. $L 1$ norm estimator}

A popular family of robust estimators is the L1 norm estimators $(\rho(x)=\|x\|)$ that are used in super-resolution problem [52-55]. We rewrite the definition of these estimators in the super-resolution context as the following minimization problem:

$$
\underline{X}=\underset{\underline{X}}{\operatorname{ArgMin}}\left\{\sum_{k=1}^{N}\left\|D_{k} H_{k} F_{k} \underline{X}-\underline{Y}_{k}\right\|\right\} .
$$

The L1 norm is not sensitive to outliers because the influence function, $\rho^{\prime}(\cdot)$, is constant and bounded but the L1 norm produces an estimator with higher variance than the optimal L2 (quadratic) norm. The L1 norm function $(\rho(\cdot))$ and its influence function $\left(\rho^{\prime}(\cdot)\right)$ are shown in Figures 2(a-1) and $2(\mathrm{a}-2)$, respectively.

\subsection{L2 norm estimator}

Another popular family of estimators is the L2 norm estimators that are used in super-resolution problem $[28,29$, 45-49]. We rewrite the definition of these estimators in the super-resolution context as the following minimization problem:

$$
\underline{X}=\underset{\underline{X}}{\operatorname{ArgMin}}\left\{\sum_{k=1}^{N}\left\|D_{k} H_{k} F_{k} \underline{X}-\underline{Y}_{k}\right\|_{2}^{2}\right\} .
$$

The L2 norm produces estimator with lower variance than the optimal L1 norm, but the L2 norm is very sensitive to outliers because the influence function increases linearly and without bound. The L2 norm function $(\rho(\cdot))$ and its influence function $\left(\rho^{\prime}(\cdot)\right)$ are shown in Figures $2(\mathrm{~b}-1)$ and 2(b-2), respectively. 


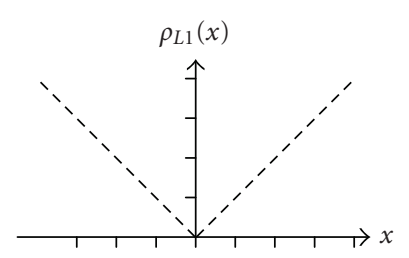

(a-1) L1 norm function

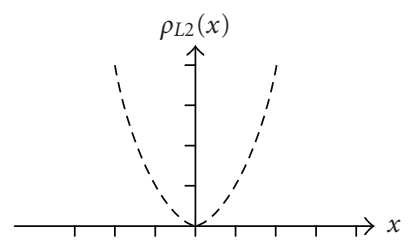

(b-1) L2 norm function

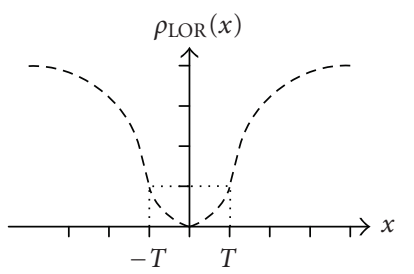

(c-1) Lorentzian norm function

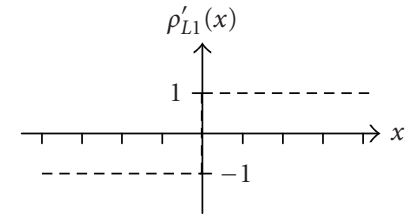

(a-2) L1 norm influence function

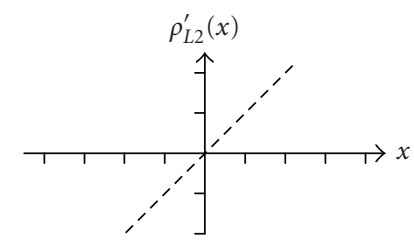

(b-2) L2 norm influence function

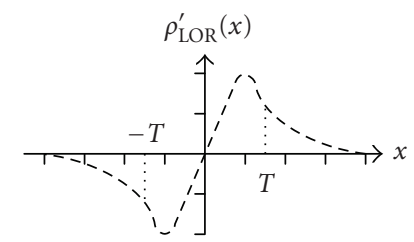

(c-2) Lorentzian norm influence function

FIgURE 2: The norm function and the influence function.

\subsection{Robust norm estimator}

A robust estimation is an estimated technique that is resistant to such outliers. In SRR framework, outliers are measured images or corrupted images that are highly inconsistent with the high-resolution original image. Outliers may arise from several reasons such as procedural measurement error, noise and inaccurate mathematical model. Outliers should be investigated carefully, therefore we need to analyze the outlier in a way which minimizes their effect on the estimated model. L2 norm estimation is highly susceptible to even small numbers of discordant observations or outliers. For L2 norm estimation, the influence of the outlier is much larger than the other measured data because L2 norm estimation weights the error quadraticly. Consequently, the robustness of L2 norm estimation is poor.

Much can be improved if the influence is bounded in one way or another. This is exactly the general idea of applying a robust error norm. Instead of using the sum of squared differences (4), this error norm should be selected such that above a given level of $x$ its influence is ruled out. In addition, one would like to have $\rho(x)$ being smooth so that numerical minimization of (5) is not too difficult. The suitable choice (among others) is so-called Lorentzian error norm [65-68] that is defined in (6). We rewrite the definition of these estimators in the super-resolution context as the following minimization problem:

$$
\begin{gathered}
\underline{X}=\underset{\underline{X}}{\operatorname{ArgMin}}\left\{\sum_{k=1}^{N} \rho_{\mathrm{LOR}}\left(D_{k} H_{k} F_{k} \underline{X}-\underline{Y}_{k}\right)\right\}, \\
\rho_{\mathrm{LOR}}(x)=\log \left[1+\frac{1}{2}\left(\frac{x}{T}\right)^{2}\right] .
\end{gathered}
$$

The parameter $T$ is Lorentzian constant parameter that is a soft threshold value. For values of $x$ smaller than $T$, the function follows the L2 norm. For values larger than $T$, the function gets saturated. Consequently, for small values of $x$, the derivative of $\rho^{\prime}(x)=\partial\{\rho(x)\} / \partial x$ of $\rho(x)$ is nearly a constant. But for large values of $x$ (for outliers), it becomes nearly zero. Therefore, in a Gauss-Newton style of optimization, the Jacobian matrix is virtually zero for outliers. Only residuals that are about as large as $T$ or smaller than that play a role.

From L1 and L2 norm estimation point of view, Lorentzian's norm is equivalent to the L1 norm for large 
value. But for normally distributed data, the L1 norm produces estimates with higher variance than the optimal L2 (quadratic) norm, so Lorentzian's norm is designed to be quadratic for small values. The Lorentzian norm function $(\rho(\cdot))$ and its influence function $\left(\rho^{\prime}(\cdot)\right)$ are shown in Figures 2(c-1) and 2(c-2), respectively.

\section{ROBUST SUPER-RESOLUTION RECONSTRUCTION}

This section proposes the robust SRR using L1, L2, and Lorentzian norm minimization with different regularization functions. Typically, super-resolution reconstruction is an inverse problem [45-49] thus the process of computing an inverse solution can be, and often is, extremely unstable in that a small change in measurement (such as noise) can lead to an enormous change in the estimated image (SR image). Therefore, super-resolution reconstruction is an ill-posed or ill-condition problem. An important point is that it is commonly possible to stabilize the inversion process by imposing additional constraints that bias the solution, a process that is generally referred to as regularization. Regularization is frequently essential to produce a usable solution to an otherwise intractable ill-posed or ill-conditioned inverse problem. Hence, considering regularization in super-resolution algorithm as a means for picking a stable solution is very useful, if not necessary. Also, regularization can help the algorithm to remove artifacts from the final answer and improve the rate of convergence.

\subsection{L1 norm SRR with Laplacian regularized function [53]}

A regularization term compensates the missing measurement information with some general prior information about the desirable HR solution, and is usually implemented as a penalty factor in the generalized minimization cost function. From (3), we rewrite the definition of these estimators in the super-resolution context as the following minimization problem:

$$
\underline{X}=\underset{\underline{X}}{\operatorname{ArgMin}}\left\{\sum_{k=1}^{N}\left\|D_{k} H_{k} F_{k} \underline{X}-\underline{Y}_{k}\right\|+\lambda \cdot \Upsilon(\underline{X})\right\} .
$$

In general, Tikhonov regularization $\Upsilon(\cdot)$ was replaced by matrix realization of the Laplacian kernel [53], the most classical and simplest regularization cost function, and where the Laplacian kernel is defined as

$$
\Gamma=\frac{1}{8}\left[\begin{array}{ccc}
1 & 1 & 1 \\
1 & -8 & 1 \\
1 & 1 & 1
\end{array}\right]
$$

Combining the Laplacian regularization, we propose the solution of the super-resolution problem as follows:

$$
\underline{X}=\underset{\underline{X}}{\operatorname{ArgMin}}\left\{\sum_{k=1}^{N}\left\|D_{k} H_{k} F_{k} \underline{X}-\underline{Y}_{k}\right\|+\lambda \cdot(\Gamma \underline{X})^{2}\right\} .
$$

By the steepest descent method, the solution of problem (9) is defined as

$$
\begin{aligned}
\underline{\hat{X}}_{n+1}=\underline{\hat{X}}_{n}+\beta \cdot\{ & \left(\sum_{k=1}^{N} G_{k}^{T} H_{k}^{T} D_{k}^{T} \operatorname{sign}\left(D_{k} H_{k} G_{k} \underline{\hat{X}}_{n}-\underline{Y}_{k}\right)\right) \\
& \left.-\left(\lambda \cdot\left(\Gamma^{T} \Gamma\right) \underline{\hat{X}}_{n}\right)\right\},
\end{aligned}
$$

where $\beta$ is a scalar defining the step size in the direction of the gradient.

\subsection{L1 norm SRR with BTV (Bitotal variance) function [52-55]}

A robust regularization function called bilateral-TV (BTV) was introduced in $[51,53]$, therefore the BTV regularization is defined as

$$
\Upsilon(X)=\sum_{l=-P}^{P} \sum_{m=0}^{P} \alpha^{|m|+|l|}\left\|\underline{X}-S_{x}^{l} S_{y}^{m} \underline{X}\right\|,
$$

where matrices (operators) $S_{x}^{l}$ and $S_{y}^{m}$ shift $\underline{X}$ by $l$ and $m$ pixels in horizontal and vertical directions, respectively, presenting several scales of derivatives. The scalar weight $\alpha, 0<\alpha<$ 1 , is applied to give a spatially decaying effect to the summation of the regularization terms $[51,53]$. Combining the BTV regularization, we rewrite the definition of these estimators in the super-resolution context as the following minimization problem:

$$
\begin{aligned}
\underline{X}=\underset{\underline{X}}{\operatorname{ArgMin}}\left\{\sum_{k=1}^{N}(\right. & \|D \cdot H \cdot G(k) \cdot \underline{X}(t)-\underline{Y}(k)\| \\
& \left.\left.+\lambda\left(\sum_{l=-P}^{P} \sum_{m=0}^{P} \alpha^{|m|+|l|}\left\|\underline{X}-S_{x}^{l} S_{y}^{m} \underline{X}\right\|\right)\right)\right\} .
\end{aligned}
$$

By the steepest descent method, the solution of problem (13) is defined as

$$
\begin{gathered}
\underline{\hat{X}}_{n+1}(t) \\
=\underline{\hat{X}}_{n}(t)+\beta \cdot\left\{\left(\sum_{k=1}^{N} G_{k}^{T} H_{k}^{T} D_{k}^{T} \operatorname{sign}\left(D_{k} H_{k} G_{k} \underline{\hat{X}}_{n}-\underline{Y}_{k}\right)\right)\right. \\
-\lambda\left(\sum_{l=-P}^{P} \sum_{m=0}^{P} \alpha^{|m|+|l|}\left(I-S_{x}^{l} S_{y}^{m}\right)\right. \\
\left.\left.\cdot \operatorname{sign}\left(\underline{\hat{X}}-S_{x}^{l} S_{y}^{m} \underline{\hat{X}}\right)\right)\right\} .
\end{gathered}
$$




\subsection{L2 norm SRR with Laplacian regularized function $[28,29]$}

From (4), we rewrite the definition of these estimators in the super-resolution context as the following minimization problem:

$$
\underline{X}=\underset{\underline{X}}{\operatorname{ArgMin}}\left\{\sum_{k=1}^{N}\left\|D_{k} H_{k} F_{k} \underline{X}-\underline{Y}_{k}\right\|_{2}^{2}\right\} .
$$

Combining the Laplacian regularization, we propose the solution of the super-resolution problem as follows:

$$
\begin{aligned}
& \underline{X}=\underset{\underline{X}}{\operatorname{ArgMin}}\left\{\sum_{k=1}^{N}\left\|D_{k} H_{k} F_{k} \underline{X}-\underline{Y}_{k}\right\|_{2}^{2}+\lambda \cdot \Upsilon(\underline{X})\right\}, \\
& \underline{X}=\underset{\underline{X}}{\operatorname{ArgMin}}\left\{\sum_{k=1}^{N}\left\|D_{k} H_{k} F_{k} \underline{X}-\underline{Y}_{k}\right\|_{2}^{2}+\lambda \cdot(\Gamma \underline{X})^{2}\right\} .
\end{aligned}
$$

By the steepest descent method, the solution of problem (16) is defined as

$$
\begin{aligned}
\underline{\hat{X}}_{n+1}=\underline{\hat{X}}_{n}+\beta \cdot\{ & \sum_{k=1}^{N} F_{k}^{T} H_{k}^{T} D_{k}^{T}\left(\underline{Y}_{k}-D_{k} H_{k} F_{k} \underline{\hat{X}}_{n}\right) \\
& \left.-\left(\lambda \cdot\left(\Gamma^{T} \Gamma\right) \underline{\hat{X}}_{n}\right)\right\} .
\end{aligned}
$$

\subsection{L2 norm SRR with BTV (Bitotal variance) function [52-55]}

Combining the BTV regularization, we propose the solution of the super-resolution problem as follows:

$$
\begin{aligned}
\underline{X}=\underset{\underline{X}}{\operatorname{ArgMin}}\left\{\sum_{k=1}^{N}(\right. & \|D \cdot H \cdot G(k) \cdot \underline{X}(t)-\underline{Y}(k)\|_{2}^{2} \\
& \left.\left.+\lambda\left(\sum_{l=-P}^{P} \sum_{m=0}^{P} \alpha^{|m|+|l|}\left\|\underline{X}-S_{x}^{l} S_{y}^{m} \underline{X}\right\|\right)\right)\right\} .
\end{aligned}
$$

By the steepest descent method, the solution of problem (18) is defined as

$$
\begin{gathered}
\underline{\hat{X}}_{n+1}(t)=\underline{\hat{X}}_{n}(t)+\beta \cdot\left\{\left(\sum_{k=1}^{N} F_{k}^{T} H_{k}^{T} D_{k}^{T}\left(\underline{Y}_{k}-D_{k} H_{k} F_{k} \underline{\hat{X}}_{n}\right)\right)\right. \\
-\lambda\left(\sum_{l=-P}^{P} \sum_{m=0}^{P} \alpha^{|m|+|l|}\left(I-S_{x}^{l} S_{y}^{m}\right)\right. \\
\left.\left.\cdot \operatorname{sign}\left(\underline{\hat{X}}-S_{x}^{l} S_{y}^{m} \underline{\hat{X}}\right)\right)\right\} .
\end{gathered}
$$

\subsection{Lorentzian norm SRR with Laplacian regularized function [69]}

In this section, we propose the novel robust SRR using Lorentzian error norm. From (5), we rewrite the definition of these robust estimators in the super-resolution context as the following minimization problem:

$$
\begin{gathered}
\underline{X}=\underset{\underline{X}}{\operatorname{ArgMin}}\left\{\sum_{k=1}^{N} \rho_{\mathrm{LOR}}\left(D_{k} H_{k} F_{k} \underline{X}-\underline{Y}_{k}\right)\right\}, \\
\rho_{\mathrm{LOR}}(x)=\log \left[1+\frac{1}{2}\left(\frac{x}{T}\right)^{2}\right] .
\end{gathered}
$$

Combining the Laplacian regularization, we propose the solution of the super-resolution problem as follows:

$$
\begin{aligned}
& \underline{X}=\underset{\underline{X}}{\operatorname{ArgMin}}\left\{\sum_{k=1}^{N} \rho_{\mathrm{LOR}}\left(D_{k} H_{k} F_{k} \underline{X}-\underline{Y}_{k}\right)+\lambda \cdot \Upsilon(\underline{X})\right\}, \\
& \underline{X}=\underset{\underline{X}}{\operatorname{ArgMin}}\left\{\sum_{k=1}^{N} \rho_{\mathrm{LOR}}\left(D_{k} H_{k} F_{k} \underline{X}-\underline{Y}_{k}\right)+\lambda \cdot(\Gamma \underline{X})^{2}\right\} .
\end{aligned}
$$

By the steepest descent method, the solution of problem (22) is defined as

$$
\begin{gathered}
\underline{\hat{X}}_{n+1}=\underline{\hat{X}}_{n}+\beta \cdot\left\{\sum_{k=1}^{N} F_{k}^{T} H_{k}^{T} D_{k}^{T} \cdot \rho_{\mathrm{LOR}}^{\prime}\left(\underline{Y}_{k}-D_{k} H_{k} F_{k} \underline{\hat{X}}_{n}\right)\right. \\
\left.-\left(\lambda \cdot\left(\Gamma^{T} \Gamma\right) \underline{\hat{X}}_{n}\right)\right\} \\
\rho_{\mathrm{LOR}}^{\prime}(x)=\frac{2 x}{2 T^{2}+x^{2}}
\end{gathered}
$$

\subsection{Lorentzian norm SRR with Lorentzian-Laplacian regularized function [69]}

Combining the Lorentzian-Laplacian regularization, we propose the solution of the super-resolution problem as follows:

$$
\underline{X}=\underset{\underline{X}}{\operatorname{ArgMin}}\left\{\sum_{k=1}^{N} \rho_{\mathrm{LOR}}\left(D_{k} H_{k} F_{k} \underline{X}-\underline{Y}_{k}\right)+\lambda \cdot \psi_{\mathrm{LOR}}(\Gamma \underline{X})\right\},
$$

$$
\psi_{\mathrm{LOR}}(x)=\log \left[1+\frac{1}{2}\left(\frac{x}{T_{g}}\right)^{2}\right]
$$

By the steepest descent method, the solution of problem (25) is defined as

$$
\begin{gathered}
\underline{\hat{X}}_{n+1}=\underline{\hat{X}}_{n}+\beta \cdot\left\{\sum_{k=1}^{N} F_{k}^{T} H_{k}^{T} D_{k}^{T} \cdot \rho_{\mathrm{LOR}}^{\prime}\left(\underline{Y}_{k}-D_{k} H_{k} F_{k} \underline{\hat{X}}_{n}\right)\right. \\
\left.-\left(\lambda \cdot \Gamma^{T} \cdot \psi_{\mathrm{LOR}}^{\prime}\left(\Gamma \underline{\hat{X}}_{n}\right)\right)\right\} \\
\psi_{\mathrm{LOR}}^{\prime}(x)=\frac{2 x}{2 T_{g}^{2}+x^{2}}
\end{gathered}
$$




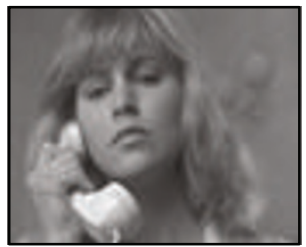

(a-1,,, $\mathrm{m}-1)$ Original HR image (Frame 40)
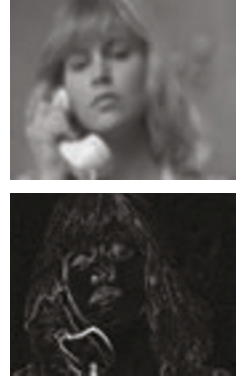

(a-2) Corrupted LR image (noiseless)

( $\mathrm{PSNR}=$

$32.1687 \mathrm{~dB}$ )
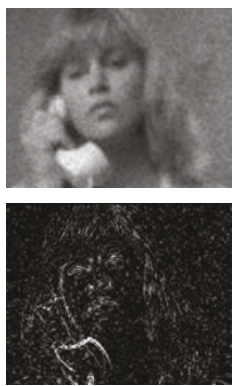

(b-2) Corrupted LR image

(AWGN:SNR = $25 \mathrm{~dB})(\mathrm{PSNR}=$ $30.1214 \mathrm{~dB}$ )
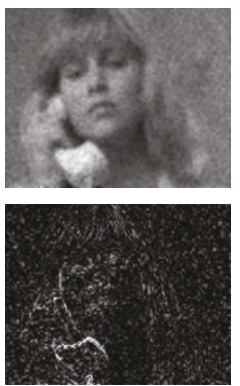

(c-2) Corrupted LR image (AWGN:SNR = $22.5 \mathrm{~dB})(\mathrm{PSNR}=$ $29.0233 \mathrm{~dB}$ )
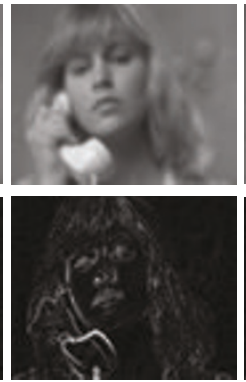

(a-3) L1 SRR image with Lap reg. $(\mathrm{PSNR}=$ $32.1687 \mathrm{~dB})$ $(\beta=1, \lambda=0)$
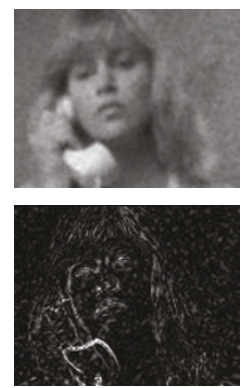

(b-3) L1 SRR image with Lap reg. $(\mathrm{PSNR}=$ $30.3719 \mathrm{~dB})$ $(\beta=0.5, \lambda=1)$
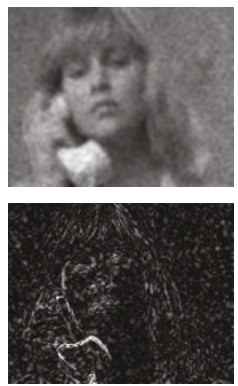

(c-3) L1 SRR image with Lap reg. $(\mathrm{PSNR}=$ $29.6481 \mathrm{~dB})$ $(\beta=0.5, \lambda=1)$
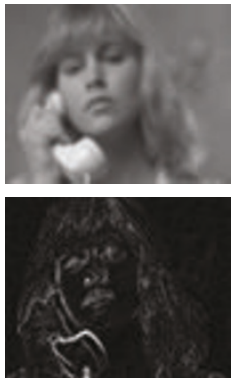

(a-4) L1 SRR image with BTV reg. $(\mathrm{PSNR}=$ $32.1687 \mathrm{~dB})$ $(\beta=1, \lambda=$ $0, P=1$, $\alpha=0.7$ )
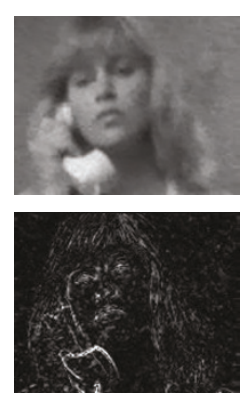

(b-4) L1 SRR image with BTV reg. $(\mathrm{PSNR}=$ $30.3295 \mathrm{~dB})$ $(\beta=0.5$, $\lambda=0.4$, $P=2, \alpha=0.7)$
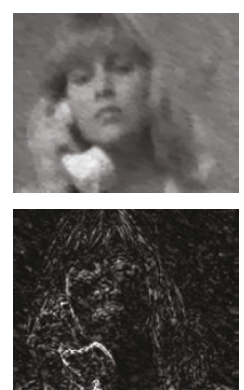

(c-4) L1 SRR image with BTV reg. $(\mathrm{PSNR}=$ $29.5322 \mathrm{~dB})$ $(\beta=0.5$, $\lambda=0.4$, $P=1, \alpha=0.7$ )

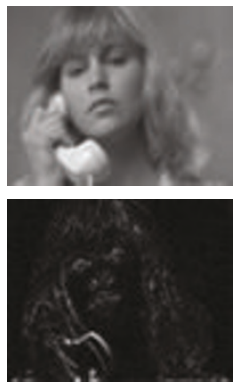

(a-5) L2 SRR image with Lap reg. $(\mathrm{PSNR}=$ $34.2 \mathrm{~dB})$ $(\beta=1, \lambda=0)$
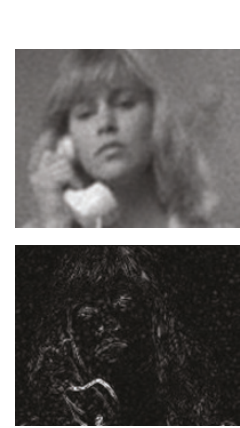

(b-5) L2 SRR image with Lap reg. (PSNR = $32.3688 \mathrm{~dB})$ $(\beta=0.5, \lambda=1)$

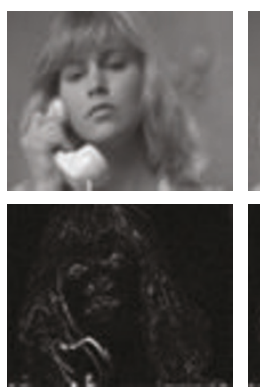

(a-6) L2 SRR image with BTV reg. $(\mathrm{PSNR}=$ $34.2 \mathrm{~dB})$ $(\beta=1, \lambda=$ $0, P=1$, $\alpha=0.7$ )
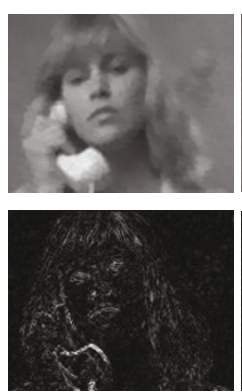

(b-6) L2 SRR image with BTV reg. $(\mathrm{PSNR}=$ $32.1643 \mathrm{~dB})$ $(\beta=0.5, \lambda=$ $0.4, P=1$, $\alpha=0.7)$
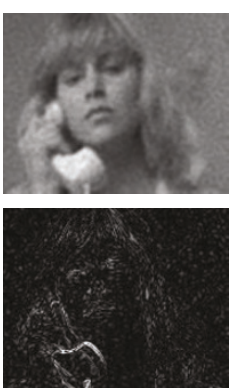

\section{(c-5) L2 SRR}

image with Lap reg. $(\mathrm{PSNR}=$

$31.6384 \mathrm{~dB})$

$(\beta=1, \lambda=1)$
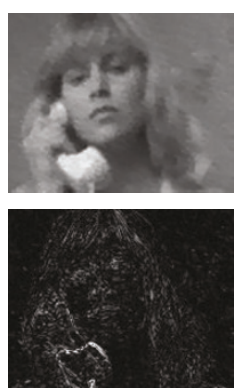

(c-6) L2 SRR

image with BTV reg. (PSNR $=$

$31.5935 \mathrm{~dB})$

$(\beta=0.5$,

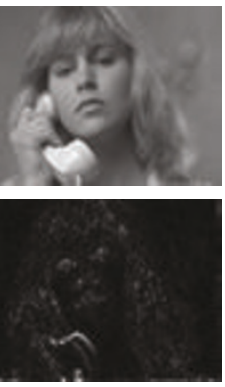

(a-7) Lor. SRR reg. $(\mathrm{PSNR}=$ $35.2853 \mathrm{~dB})$ $(\beta=0.25$,
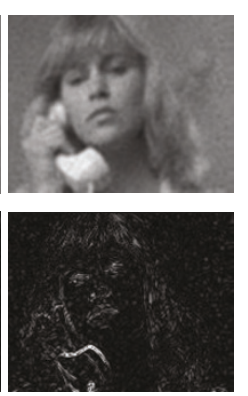

$\lambda=0.4$,

$P=1, \alpha=0.7$ ) image with Lap $\lambda=0, T=3$ )

(b-7) Lor. SRR image with Lap reg. $(\mathrm{PSNR}=$ $32.2341 \mathrm{~dB})$ $(\beta=0.5, \lambda=1$, $T=9)$

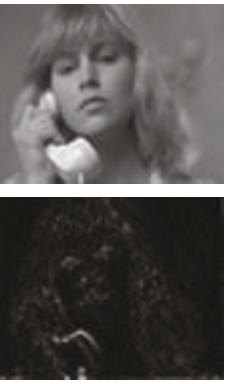

(a-8) Lor. SRR image with Lor-Lap reg. $(\mathrm{PSNR}=$ $35.2853 \mathrm{~dB}$ ) $(\beta=0.25, \lambda=0$, $T=1, T_{g}=1$ )
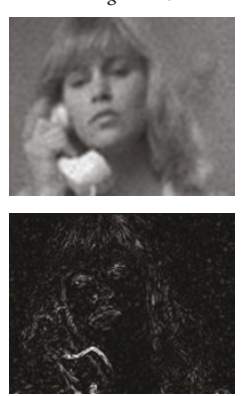

(b-8) Lor. SRR image with

Lor.-Lap reg. $(\mathrm{PSNR}=$

$32.3591 \mathrm{~dB})$

$(\beta=0.5$,

$\lambda=0.75$,

$\left.T=9, T_{g}=3\right)$
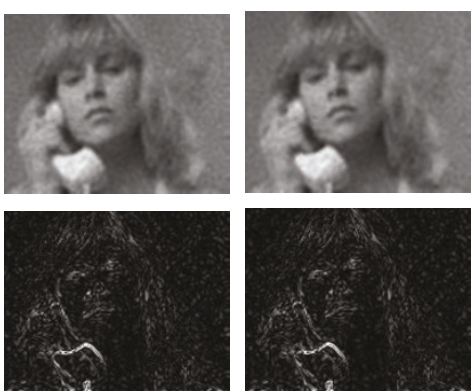

(c-7) Lor. SRR image with Lap reg. $(\mathrm{PSNR}=$ $31.4751 \mathrm{~dB})$ $(\beta=0.5$, $\lambda=1, T=9)$

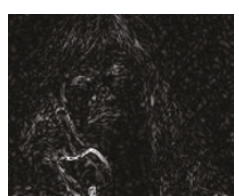

(c-8) Lor. SRR image with Lor.-Lap reg. $(\mathrm{PSNR}=$ $31.6169 \mathrm{~dB})$ $(\beta=0.5, \lambda=1$, $T=9, T_{g}=3$ )

Figure 3: The experimental results of proposed method. 


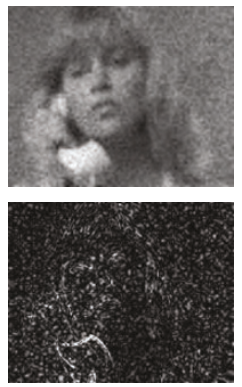

(d-2) Corrupted

LR image

(AWGN:SNR = $20 \mathrm{~dB})(\mathrm{PSNR}=$ $27.5316 \mathrm{~dB}$ )
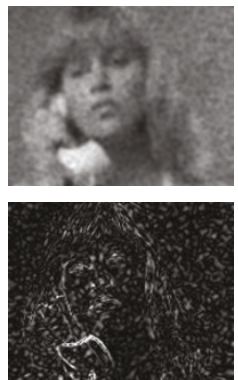

(d-3) L1 SRR image with Lap reg. $(\mathrm{PSNR}=$ $28.7003 \mathrm{~dB})$ $(\beta=0.5, \lambda=1)$
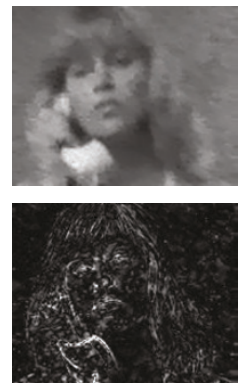

(d-4) L1 SRR image with BTV reg. $(\mathrm{PSNR}=$ $28.9031 \mathrm{~dB})$ $(\beta=0.5$, $\lambda=0.4, P=2$, $\alpha=0.7)$
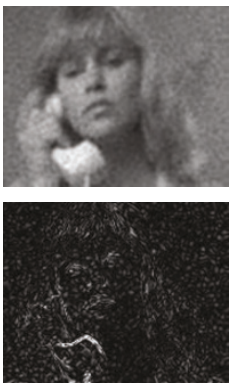

(d-5) L2 SRR image with Lap reg. $(\mathrm{PSNR}=$ $30.6898 \mathrm{~dB})$ $(\beta=0.5, \lambda=1)$
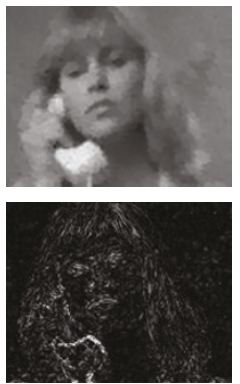

(d-6) L2 SRR image with BTV reg. $(\mathrm{PSNR}=$ $31.0056 \mathrm{~dB})$

$(\beta=0.5$,

$\lambda=0.3, P=2$, $\alpha=0.7)$
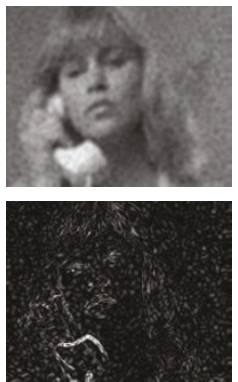

(d-7) Lor. SRR image with Lap reg. $(\mathrm{PSNR}=$ $30.5472 \mathrm{~dB}$ ) $(\beta=0.5$, $\lambda=1, T=9)$
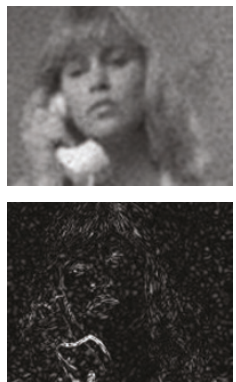

(d-8) Lor. SRR image with Lor.-Lap reg. (PSNR = $30.7486 \mathrm{~dB})$ $(\beta=0.5, \lambda=1$, $\left.T=9, T_{g}=5\right)$
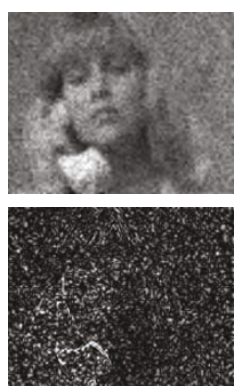

(e-2) Corrupted LR image (AWGN:SNR = $17.5 \mathrm{~dB})(\mathrm{PSNR}=$ $25.7332 \mathrm{~dB}$ )
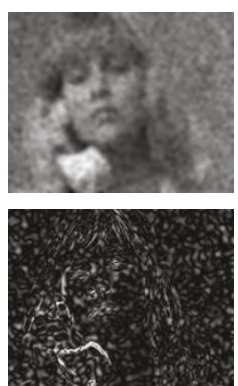

(e-3) L1 SRR image with Lap reg. $(\mathrm{PSNR}=$ $27.5771 \mathrm{~dB})$ $(\beta=1, \lambda=1)$
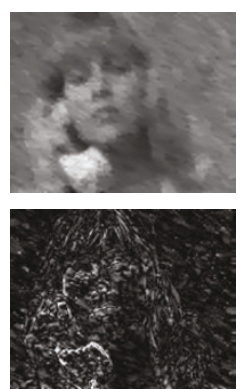

(e-4) L1 SRR image with BTV reg. $(\mathrm{PSNR}=$ $27.7575 \mathrm{~dB})$

$(\beta=0.5$, $\lambda=0.5, P=1$, $\alpha=0.7)$
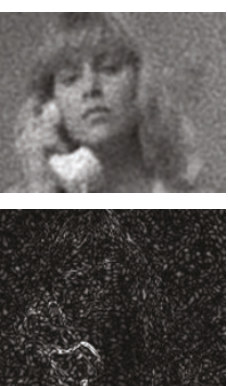

(e-5) L2 SRR image with Lap reg. $(\mathrm{PSNR}=$ $29.3375 \mathrm{~dB})$ $(\beta=0.5, \lambda=1)$
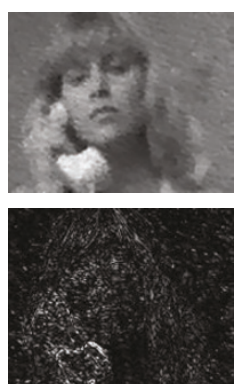

(e-6) L2 SRR image with BTV reg. (PSNR $=$ $29.4085 \mathrm{~dB})$

$(\beta=0.5$, $\lambda=0.5, P=1$, $\alpha=0.7$ )
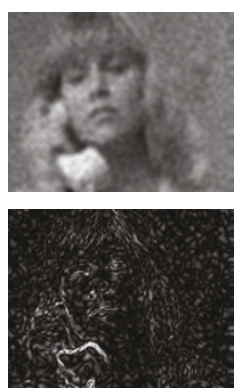

(e-7) Lor. SRR image with Lap reg. $(\mathrm{PSNR}=$ $29.4712 \mathrm{~dB}$ ) $(\beta=0.5, \lambda=1$, $T=5)$
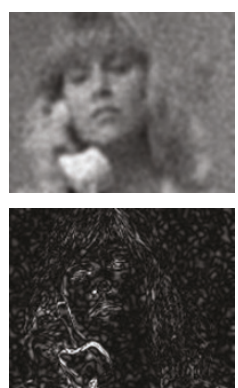

(e-8) Lor. SRR image with Lor.-Lap reg. (PSNR = $29.691 \mathrm{~dB})$ $(\beta=0.5, \lambda=1$, $T=5, T_{g}=5$ )
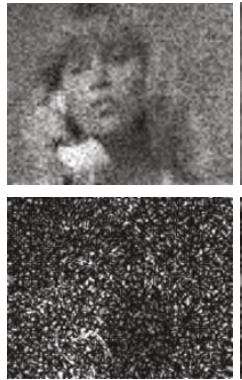

(f-2) Corrupted

LR image

(AWGN:SNR = $15 \mathrm{~dB})(\mathrm{PSNR}=$ $23.7086 \mathrm{~dB}$ )
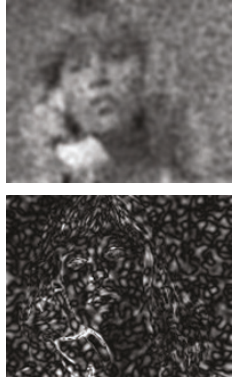

(f-3) L1 SRR image with Lap reg. $(\mathrm{PSNR}=$ $26.2641 \mathrm{~dB})$ $(\beta=0.5, \lambda=1)$
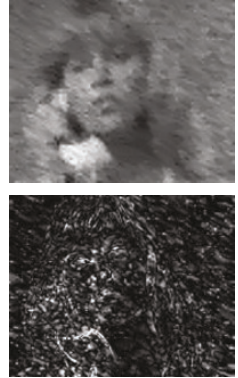

(f-4) L1 SRR image with BTV reg. $(\mathrm{PSNR}=$ $26.9064 \mathrm{~dB})$ ( $\beta=0.5$, $\lambda=0.8, P=1$, $\alpha=0.7$ )
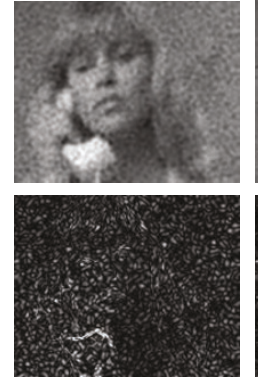

(f-5) L2 SRR image with Lap reg. $(\mathrm{PSNR}=$ $27.6671 \mathrm{~dB})$ $(\beta=0.5, \lambda=1)$
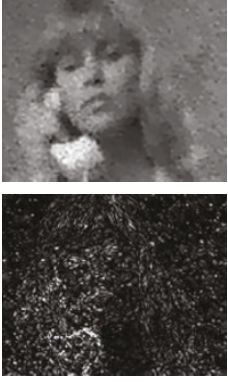

(f-6) L2 SRR image with BTV reg. $(\mathrm{PSNR}=$ $27.8418 \mathrm{~dB})$

( $\beta=0.5$,

$\lambda=0.3, P=2$, $\alpha=0.7)$
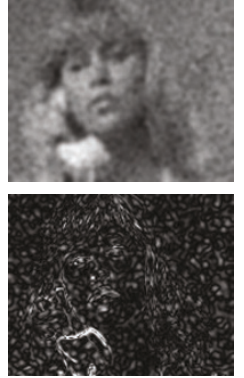

(f-7) Lor. SRR image with Lap reg. $(\mathrm{PSNR}=$ $28.1516 \mathrm{~dB})$ $(\beta=0.5, \lambda=1$, $T=5)$
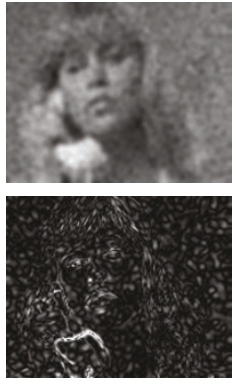

(f-8) Lor. SRR image with Lor.-Lap reg. (PSNR = $28.4389 \mathrm{~dB})$ $(\beta=0.5, \lambda=1$, $T=5, T_{g}=9$ )

Figure 3: continued. 

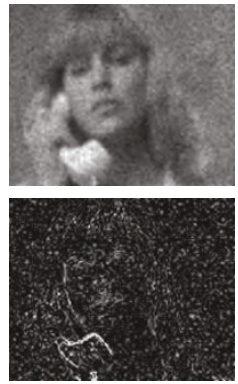

(g-2) Corrupted LR image (Poisson) (PSNR $=27.9071 \mathrm{~dB}$ )
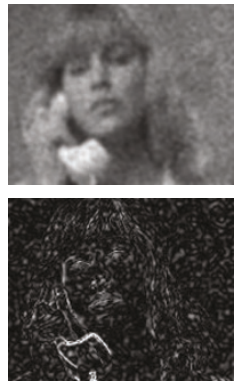

(g-3) L1 SRR image with Lap reg. $(\mathrm{PSNR}=$ $28.9197 \mathrm{~dB})$ $(\beta=1, \lambda=1)$
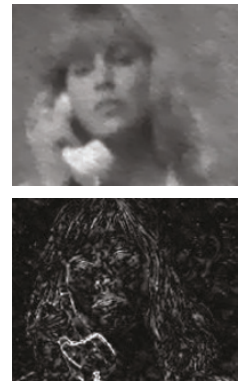

(g-4) L1 SRR image with BTV reg. $(\mathrm{PSNR}=$ $29.1201 \mathrm{~dB})$ $(\beta=0.5$, $\lambda=0.4, P=2$, $\alpha=0.7)$
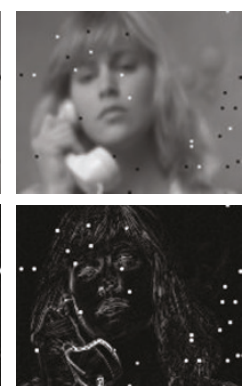

(h-4) L1 SRR image with BTV reg. $(\mathrm{PSNR}=$ $29.0649 \mathrm{~dB})$

$(\beta=1$,

$\lambda=0.5, P=2$, $\alpha=0.7$ ) $29.0649 \mathrm{~dB})$ $(\beta=1, \lambda=1)$

(h-3) L1 SRR reg. $(\mathrm{PSNR}=$
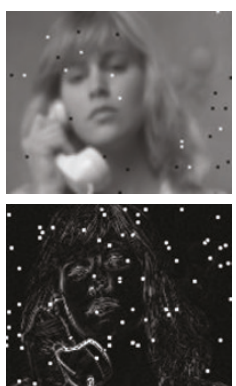

(i-2) (S\&P:D =

$0.01)$ Corrupted

LR image (PSNR $=26.4446 \mathrm{~dB}$ )
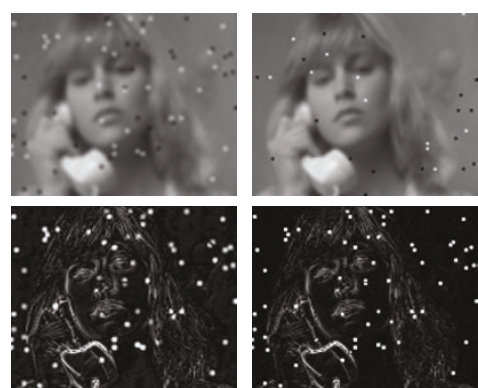

(i-3) L1 SRR image with Lap reg. $(\mathrm{PSNR}=$ $27.7593 \mathrm{~dB})$ $(\beta=1, \lambda=1)$

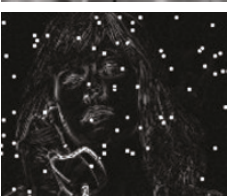

(i-4) L1 SRR image with BTV reg. $(\mathrm{PSNR}=$ $26.4446 \mathrm{~dB})$

$(\beta=1$,

$\lambda=0.5, P=1$, $\alpha=0.7$ )
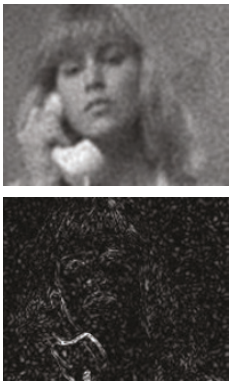

(g-5) L2 SRR image with Lap reg. $(\mathrm{PSNR}=$ $30.7634 \mathrm{~dB})$ $(\beta=0.5, \lambda=1)$
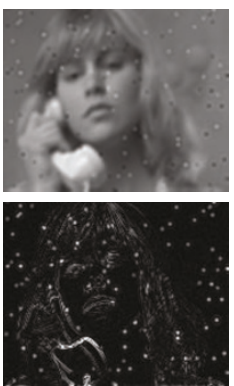

(h-5) L2 SRR image with Lap reg. $(\mathrm{PSNR}=$ $31.5021 \mathrm{~dB})$ $(\beta=0.5, \lambda=1)$
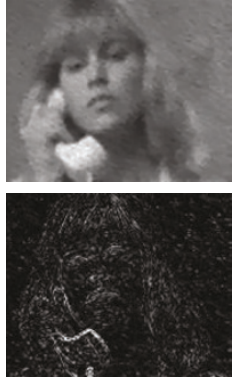

(g-6) L2 SRR image with BTV reg. $(\mathrm{PSNR}=$ $30.8631 \mathrm{~dB})$ $(\beta=0.5$, $\lambda=0.5, P=1$, $\alpha=0.7)$

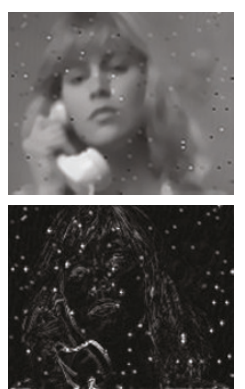

(h-6) L2 SRR image with BTV reg. $(\mathrm{PSNR}=$ $30.4617 \mathrm{~dB})$ $(\beta=0.5$, $\lambda=0.4, P=1$, $\alpha=0.7$ )

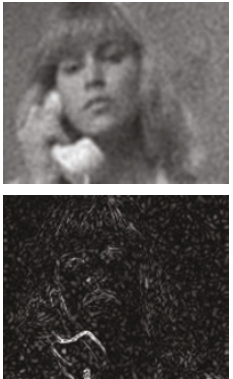

(g-7) Lor. SRR image with Lap reg. $(\mathrm{PSNR}=$ $30.6934 \mathrm{~dB})$ $(\beta=0.5, \lambda=1$, $T=9)$

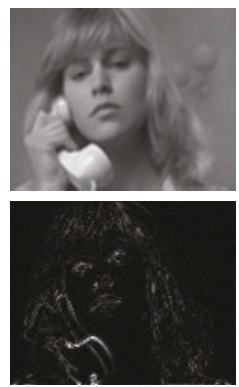

(h-7) Lor. SRR image with Lap reg. $(\mathrm{PSNR}=$ $34.7155 \mathrm{~dB})$ $(\beta=1$, $\lambda=0.25, T=9)$
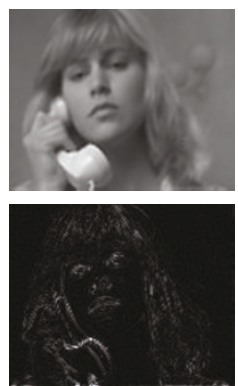

(h-8) Lor. SRR image with Lor.-Lap reg. $(\mathrm{PSNR}=$ $34.7921 \mathrm{~dB})$ $(\beta=1$, $\lambda=0.25, T=9$, $T_{g}=3$ )

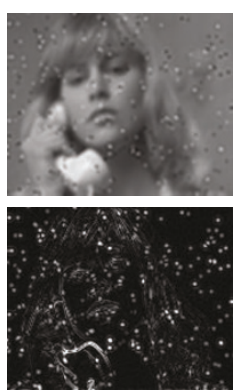

(i-5) L2 SRR image with Lap reg. $(\mathrm{PSNR}=$ $29.8395 \mathrm{~dB})$ $(\beta=0.5, \lambda=1)$

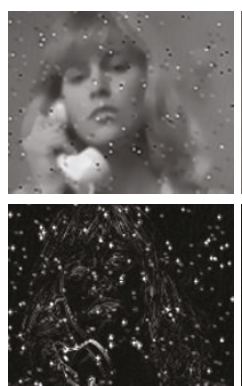

(i-6) L2 SRR image with BTV reg. $(\mathrm{PSNR}=$ $28.0337 \mathrm{~dB})$

$(\beta=0.5$,

$\lambda=0.4, P=1$, $\alpha=0.7)$

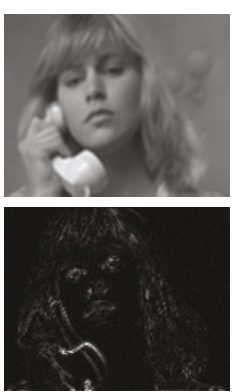

(i-7) Lor. SRR image with Lap reg. $(\mathrm{PSNR}=$ $34.7194 \mathrm{~dB})$ $(\beta=1$, $\lambda=0.25, T=5)$
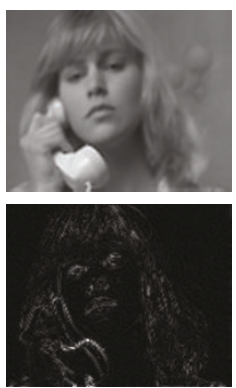

(i-8) Lor. SRR image with Lor--Lap reg. $(\mathrm{PSNR}=$ $34.7783 \mathrm{~dB})$ $(\beta=1$,

$\lambda=0.25, T=9$, $T_{g}=3$ ) 

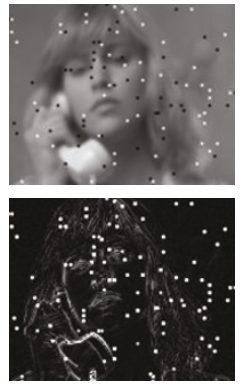

(j-2) Corrupted LR image (S\&P:D $=0.015)(\mathrm{PSNR}=$ $25.276 \mathrm{~dB}$ )
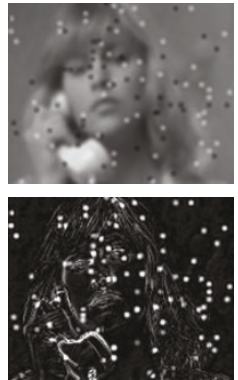

(j-3) L1 SRR image with Lap reg. (PSNR $=$ $26.9247 \mathrm{~dB})$ $(\beta=1, \lambda=1)$
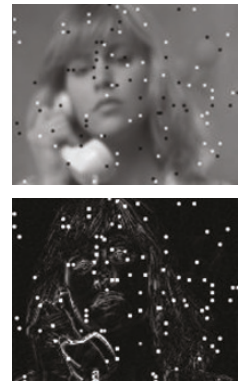

(j-4) L1 SRR image with BTV reg. $(\mathrm{PSNR}=$ $25.276 \mathrm{~dB})$ $(\beta=1$,

$\lambda=0.5, P=1$, $\alpha=0.7$ )
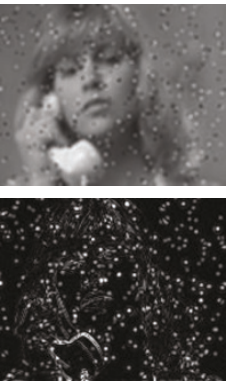

(j-5) L2 SRR image with Lap reg. $(\mathrm{PSNR}=$ $28.7614 \mathrm{~dB})$ $(\beta=0.5, \lambda=1)$
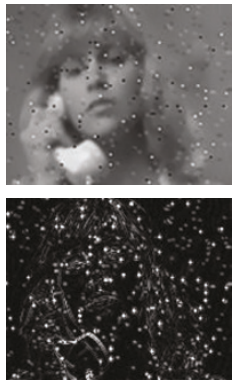

(j-6) L2 SRR image with BTV reg. (PSNR $=$ $26.8671 \mathrm{~dB})$ $(\beta=0.5$, $\lambda=0.4, P=1$, $\alpha=0.7$ )
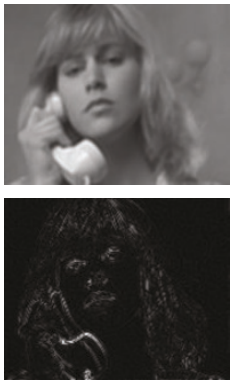

(j-7) Lor. SRR image with Lap reg. $(\mathrm{PSNR}=$ $34.6991 \mathrm{~dB})$

$(\beta=1$,

$\lambda=0.25, T=5)$
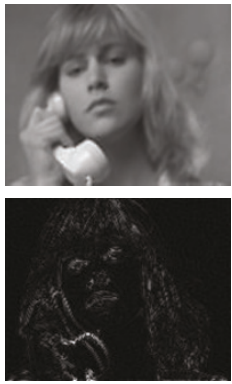

(j-8) Lor. SRR image with Lor--Lap reg. $(\mathrm{PSNR}=$ $34.7001 \mathrm{~dB})$ $(\beta=1$, $\lambda=0.25, T=9$, $T_{g}=3$ )
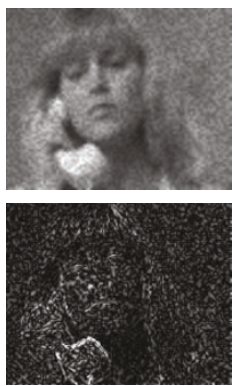

(k-2) (Speckle: $V=0.01$ ) Corrupted LR image $(\mathrm{PSNR}=$ $27.6166 \mathrm{~dB}$ )
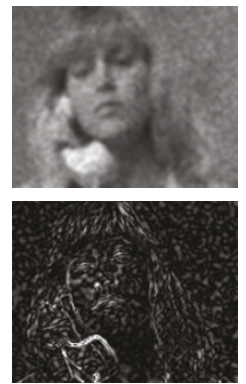

(k-3) L1 SRR image with Lap reg. $(\mathrm{PSNR}=$ $28.8289 \mathrm{~dB})$ $(\beta=0.5, \lambda=1)$
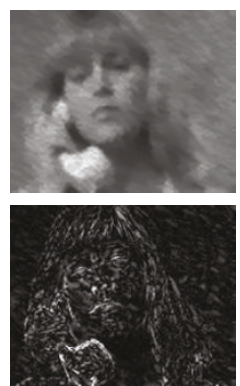

(k-4) L1 SRR image with BTV reg. $(\mathrm{PSNR}=$ $28.8656 \mathrm{~dB})$ $(\beta=0.5$, $\lambda=0.7, P=1$, $\alpha=0.7$ )

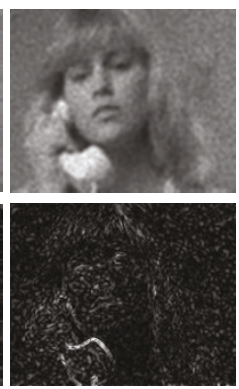

(k-5) L2 SRR image with Lap reg. $(\mathrm{PSNR}=$ $30.6139 \mathrm{~dB})$ $(\beta=0.5, \lambda=1)$
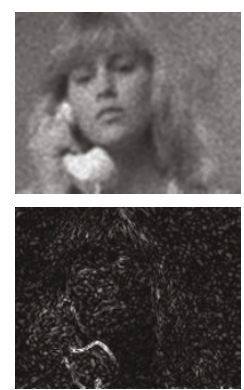

(k-6) L2 SRR image with BTV reg. $(\mathrm{PSNR}=$ $30.613 \mathrm{~dB})$ $(\beta=0.5$, $\lambda=0.5, P=1$, $\alpha=0.7$ )

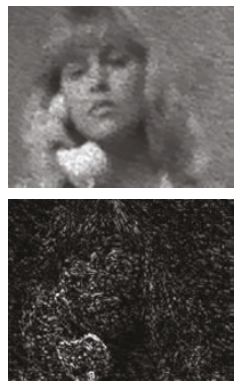

(1-6) L2 SRR image with BTV reg. $(\mathrm{PSNR}=$ $28.8859 \mathrm{~dB})$

$(\beta=0.5$,

$\lambda=0.5, P=1$, $\alpha=0.7)$
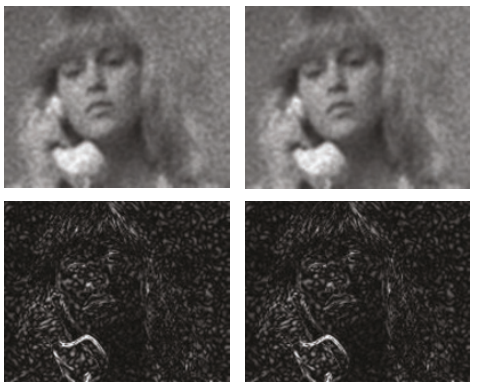

(k-7) Lor. SRR image with Lap reg. $(\mathrm{PSNR}=$ $29.8499 \mathrm{~dB})$ $(\beta=0.5, \lambda=1$, $T=9$ )

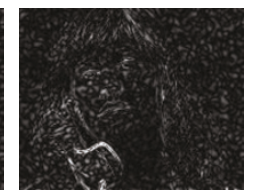

(k-8) Lor. SRR image with Lor.-Lap reg. (PSNR = $30.1287 \mathrm{~dB})$ $(\beta=0.5, \lambda=1$, $\left.T=9, T_{g}=5\right)$
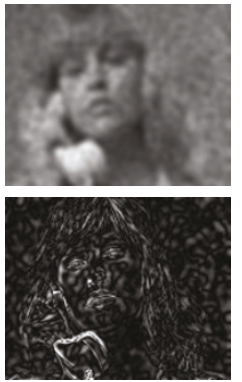

(1-7) Lor. SRR image with Lap reg. $(\mathrm{PSNR}=$ $28.5018 \mathrm{~dB})$ $(\beta=0.5, \lambda=1$, $T=1$ )

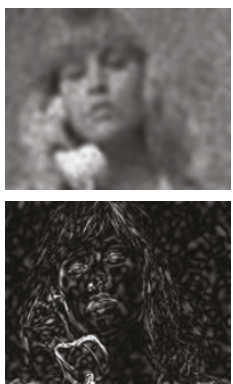

(1-8) Lor. SRR image with Lor.-Lap reg. (PSNR = $28.9779 \mathrm{~dB})$ $(\beta=0.5, \lambda=1$, $T=1, T_{g}=3$ ) 

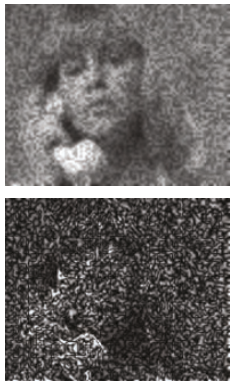

$(\mathrm{m}-2)$ (Speckle:

$V=0.03$ )

Corrupted LR

image $(\mathrm{PSNR}=$

$24.0403 \mathrm{~dB}$ )
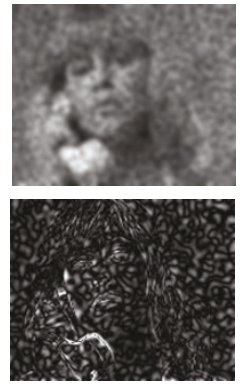

(m-3) L1 SRR image with Lap reg. (PSNR $=$ $26.8165 \mathrm{~dB})$ $(\beta=0.5, \lambda=1)$
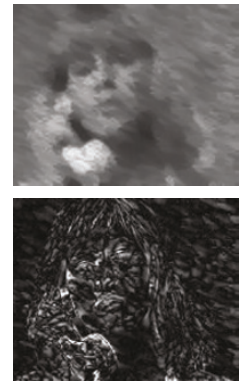

$(\mathrm{m}-4)$ L1 SRR image with BTV reg. $(\mathrm{PSNR}=$ $27.2429 \mathrm{~dB})(\beta=$ $0.5, \lambda=0.5, P=$ $1, \alpha=0.7)$
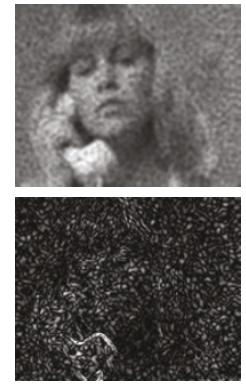

(m-5) L2 SRR image with Lap reg. $(\mathrm{PSNR}=$ $27.7654 \mathrm{~dB})$ $(\beta=0.5, \lambda=1)$
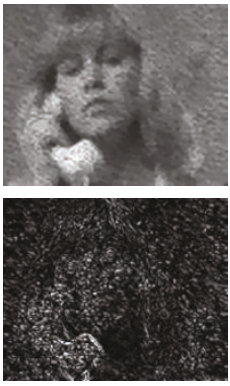

(m-6) L2 SRR image with BTV reg. $(\mathrm{PSNR}=$ $27.3751 \mathrm{~dB})$ $(\beta=0.5$, $\lambda=0.4, P=1$, $\alpha=0.7)$
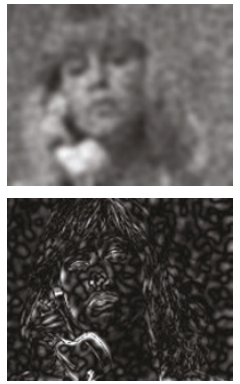

(m-7) Lor. SRR image with Lap reg. $(\mathrm{PSNR}=$ $27.9468 \mathrm{~dB})$ $(\beta=0.5, \lambda=1$, $T=1$ )

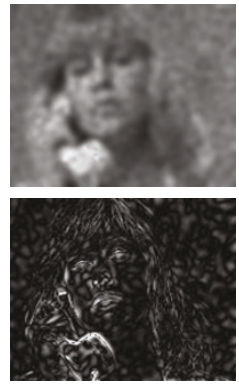

(m-8) Lor. SRR image with Lor.-Lap reg. $(\mathrm{PSNR}=$ $28.4418 \mathrm{~dB}$ ) $(\beta=0.5, \lambda=1$, $T=1, T_{g}=3$ )

Figure 3: continued.

\section{EXPERIMENTAL RESULT}

This section presents the experiments and results obtained by the proposed robust SRR methods using Lorentzian norm with Laplacian and Lorentzian-Laplacian regularizations that are calculated by (23) and (27). To demonstrate the proposed robust SRR performance, the results of L1 norm SRR with Laplacian and BTV regularizations calculated by (10) and (13) and the results of L2 norm SRR with Laplacian and BTV regularizations calculated by (17) and (19) are presented in order to compare the performance.

These experiments are implemented in MATLAB and the block size is fixed at $8 \times 8(16 \times 16$ for overlapping block $)$. In this experiment, we create a sequence of LR frames by using the 40th frame Susie sequence that is QCIF format $(176 \times 144)$ and using the Lena (standard image). First, we shifted this HR image by a pixel in the vertical direction. Then, to simulate the effect of camera PSF, this shifted image was convolved with a symmetric Gaussian low-pass filter of size $3 \times 3$ with standard deviation equal to one. The resulting image was subsampled by the factor of 2 in each direction. The same approach with different motion vectors (shifts) in vertical and horizontal directions was used to produce 4 LR images from the original scene. We added different noise model to the resulting LR frames. Next, we use 4 LR frames to generate the high-resolution image by the different SRR methods. (The criterion for parameter selection in this paper was to choose parameters which produce both most visually appealing results and highest PSNR. Therefore, to ensure fairness, each experiment was repeated several times with different parameters and the best result of each experiment was chosen [52-55].)

\subsection{Susie sequence (the 40th Frame)}

\section{Noiseless}

The original HR image is shown in Figure 3(a-1) and one of the corrupted LR images is shown in Figure 3(a-2). Next, the results of implementing the super-resolution method using L1 estimator with Laplacian regularization, L1 estimator with BTV regularization, L2 estimator with Laplacian regularization, L2 estimator with BTV regularization, Lorentzian estimator with Laplacian regularization and Lorentzian estimator with Lorentzian-Laplacian regularization are shown in Figures 3(a-3)-3(a-8), respectively. Due to noiseless effect, the results of SRR without regularization give a better result than the SRR with regularization. From the results, Lorentzian estimator can efficiently reconstruct the noiseless image than L1 and L2 estimator, about $1-3 \mathrm{~dB}$, respectively.

\section{Additive white Gaussian noise}

This experiment is 5 AWGN cases at $\mathrm{SNR}=25,22.5,20$, 17.5 , and $15 \mathrm{~dB}$, respectively, and the original HR images are shown in Figures 3(b-1)-3(f-1), respectively. The corrupted images at $\mathrm{SNR}=25,22.5,20,17.5$, and $15 \mathrm{~dB}$ are show in Figures 3(b-2)-3(f-2), respectively.

At the high SNR (SNR = 25 and $22.5 \mathrm{~dB}$ ) or low-noise power, the L2 estimator results (with Laplacian and BTV regularizations) give slightly higher PSNR than Lorentzian estimator result (with Laplacian and Lorentzian-Laplacian regularizations). However, L2 and Lorentzian estimator result have higher PSNR than L1 estimator results. At SNR $=25 \mathrm{~dB}$ and $\mathrm{SNR}=22.5 \mathrm{~dB}$, the result of $\mathrm{L} 1$ estimator with Laplacian regularization, L1 estimator with BTV regularization, L2 estimator with Laplacian regularization, L2 estimator with BTV regularization, Lorentzian estimator with Laplacian regularization, and Lorentzian estimator with Lorentzian-Laplacian regularization estimation are shown in Figures 3(b-3)-3(b8) and 3(c-3)-3(c-8), respectively.

At low SNR $(\mathrm{SNR}=20 \mathrm{~dB}, \mathrm{SNR}=17.5 \mathrm{~dB}$, and SNR $=15 \mathrm{~dB}$ ) or high-noise power, the Lorentzian estimator results (with Lorentzian-Laplacian regularization and Laplacian regularization) give the best performance than L2 estimator result (with Laplacian and BTV regularization) and L1 estimator results (with Laplacian and BTV regularization). At $\mathrm{SNR}=20 \mathrm{~dB}, \mathrm{SNR}=17.5 \mathrm{~dB}$, and $\mathrm{SNR}=15 \mathrm{~dB}$, the results 
of L1 estimator with Laplacian regularization, L1 estimator with BTV regularization, L2 estimator with Laplacian regularization, L2 estimator with BTV regularization, Lorentzian estimator with Laplacian regularization and Lorentzian estimator with Lorentzian-Laplacian regularization estimation are shown in Figures 3(d-3)-3(d-8), 3(e-3)-3(e-8), and 3(f3)-3(f-8), respectively.

From the results, the L2 estimator gives the best result for SRR estimation than Lorentzian or L1 estimator at lownoise power because the AWGN distributional characteristic is a quadratic model that is similar to L2 model. However, at high-noise power, the Lorentzian estimator will give the better result than L2 estimator since the L2 norm is very sensitive to outliers where the influence function increases linearly and without bound.

\section{Poisson noise}

The original HR image is shown in Figure 3(g-1) and one of corrupted LR images is shown in Figure 3(g-2). The Lorentzian estimator results with Lorentzian-Laplacian regularization give the highest PSNR than the Lorentzian estimator results with Laplacian regularization, L2 estimator result with Laplacian and BTV regularizations, and L1 estimator result with Laplacian and BTV regularization. The result of implementing the super-resolution method using L1 estimator with Laplacian regularization, L1 estimator with BTV regularization, L2 estimator with Laplacian regularization, L2 estimator with BTV regularization, Lorentzian estimator with Laplacian regularization and Lorentzian estimator with Lorentzian-Laplacian Regularization are shown in Figures 3(g-3)-3(g-8), respectively.

From the results, the Lorentzian estimator will give the best result than L1 and L2 estimators since the power of noise is slightly high and the distribution of noise is not quadratic model (the L2 estimator cannot estimate the no quadratic model effectively).

\section{Salt and pepper noise}

This is 3 salt and pepper noise cases at $D=0.005, D=0.01$, and $D=0.015$, respectively, and the original $\mathrm{HR}$ images are shown in Figures $3(\mathrm{~h}-1)-3(\mathrm{j}-1)$, respectively. The corrupted images at $D=0.005, D=0.01$, and $D=0.015$ are shown in Figures 3(h-2), 3(i-2), and 3(j-2), respectively. The Lorentzian estimator results (with Laplacian and LorentzianLaplacian regularizations results) give dramatically higher PSNR than L1 estimator results (with Laplacian and BTV regularization results) and L2 estimator result (with Laplacian and BTV regularizations results).

At $D=0.005, D=0.01$, and $D=0.015$, the results of L1 estimator with Laplacian regularization, L1 estimator with BTV regularization, L2 estimator with Laplacian regularization, L2 estimator with BTV regularization, Lorentzian estimator with Laplacian regularization, and Lorentzian estimator with Lorentzian-Laplacian regularization estimation are shown in Figures 3(h-3)-3(h-8), 3(i-3)-3(i-8), and 3(j4) $-3(j-8)$, respectively.
From the results, the Lorentzian estimator with Laplacian regularization and the Lorentzian estimator with LorentzianLaplacian regularization can outstandingly efficiently reconstruct the image that is corrupted by salt and pepper noise than L1 and L2 estimator about 4-5 dB. The Lorentzian estimator gives the best result for SRR estimation than L1 or L2 estimator because the Lorentzian estimator is designed to be robustness and reject outliers, the norm must be more forgiving about outliers; that is, it should increase less rapidly than $\mathrm{L} 2$.

\section{Speckle noise}

The last experiment is 3 speckle noise cases for 40th frame Susie sequence at $V=0.01, V=0.02$, and $V=0.03$, respectively, and the original HR images are shown in Figures $3(\mathrm{k}$ 1)-3(m-1), respectively. The corrupted images at $V=0.01$, $V=0.02$, and $V=0.03$ are showen in Figures $3(\mathrm{k}-2), 3(\mathrm{l}-2)$ and $3(\mathrm{~m}-2)$, respectively.

At low-noise power $(V=0.01)$, the L2 estimator results (with Laplacian and BTV regularizations) give slightly higher PSNR than Lorentzian estimator results (with Laplacian and Lorentzian-Laplacian regularizations). However, L2 and Lorentzian estimators results have higher PSNR than L1 estimator results (with Laplacian and BTV regularizations). The results of implementing the super-resolution method using L1 estimator with Laplacian regularization, L1 estimator with BTV regularization, L2 estimator with Laplacian regularization, L2 estimator with BTV regularization, Lorentzian estimator with Laplacian regularization and Lorentzian estimator with Lorentzian-Laplacian regularization are shown in Figures 3(k-3) and 3(k-8), respectively.

At high-noise power $(V=0.02$ and $V=0.03)$, the Lorentzian estimator results (with Laplacian and LorentzianLaplacian regularizations) give the best performance than L2 estimator results (with Laplacian and BTV regularizations) and L1 estimator results (with Laplacian and BTV regularizations). At $V=0.02 \mathrm{~dB}$ and $V=0.03$, the results of $\mathrm{L} 1$ estimator with Laplacian regularization, L1 estimator with BTV regularization, L2 estimator with Laplacian regularization, L2 estimator with BTV regularization, Lorentzian estimator with Laplacian regularization, and Lorentzian estimator with Lorentzian-Laplacian regularization are shown in Figures 3(1-3)-3(1-8) and 3(m-3)-3(m-8), respectively.

From the results, the Lorentzian estimator can efficiently reconstruct the image that is corrupted by speckle noise at high-noise power than L1 and L2 estimators because Lorentzian estimator is more robust for estimation to the high-power outlier than L1 and L2 estimators.

\subsection{Lena (the standard image)}

\section{Noiseless}

The original HR image is shown in Figure 4(a-1) and one of the corrupted LR images is shown in Figure 4(a-2). Next, the results of implementing the super-resolution method using L1 estimator with Laplacian regularization, L1 estimator with BTV regularization, L2 estimator with Laplacian 


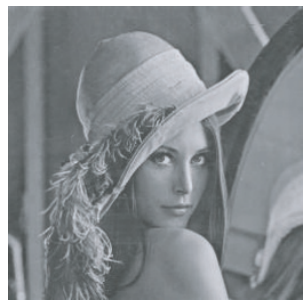

$(\mathrm{a}-1, \ldots, \mathrm{k}-1)$

Original HR image

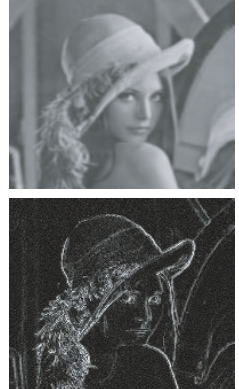

(a-2) Corrupted LR image (noiseless) (PSNR = $28.8634 \mathrm{~dB}$ )
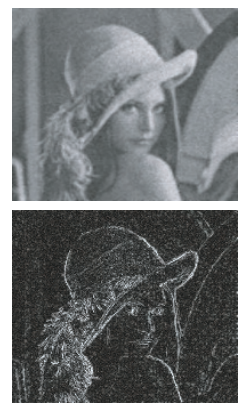

(b-2) Corrupted LR image (AWGN:SNR = $25 \mathrm{~dB})(\mathrm{PSNR}=$ $27.8884 \mathrm{~dB}$ )
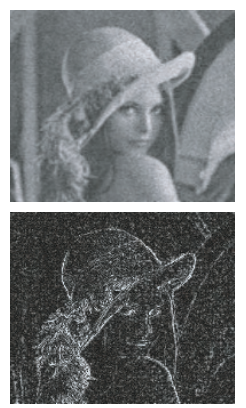

(c-2) Corrupted LR image (AWGN:SNR = $22.5 \mathrm{~dB})(\mathrm{PSNR}=$ $27.2417 \mathrm{~dB}$ )
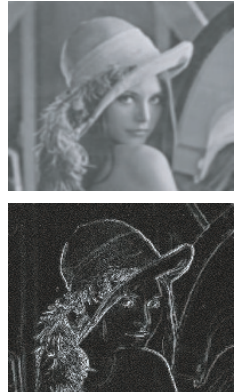

(a-3) L1 SRR image with Lap reg. $(\mathrm{PSNR}=$ $28.8634 \mathrm{~dB})$ $(\beta=1, \lambda=0)$
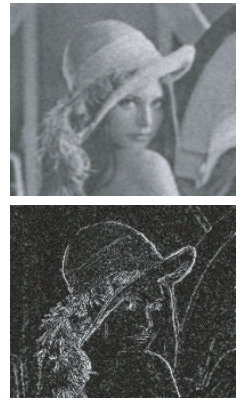

(b-3) L1 SRR image with Lap reg. $(\mathrm{PSNR}=$ $27.949 \mathrm{~dB})$ $(\beta=0.5, \lambda=1)$
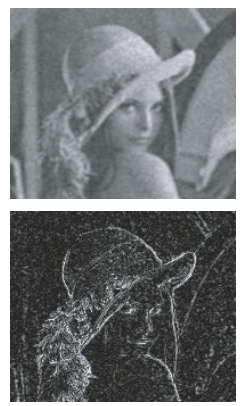

(c-3) L1 SRR image with Lap reg. $(\mathrm{PSNR}=$ $27.4918 \mathrm{~dB})$ $(\beta=0.5, \lambda=1)$
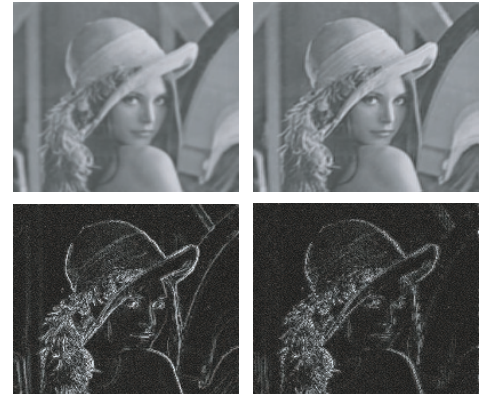

(a-4) L1 SRR image with BTV reg. $(\mathrm{PSNR}=$ $28.8634 \mathrm{~dB})$

$(\beta=1, \lambda=0$, $P=1, \alpha=0.7)$
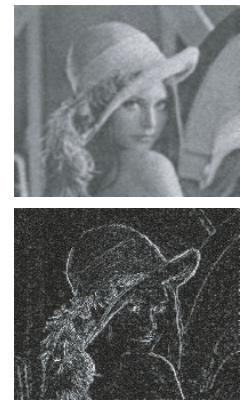

(b-4) L1 SRR image with BTV reg. $(\mathrm{PSNR}=$ $27.8884 \mathrm{~dB})$

$(\beta=0.5$,

$\lambda=0.25, P=1$, $\alpha=0.7)$
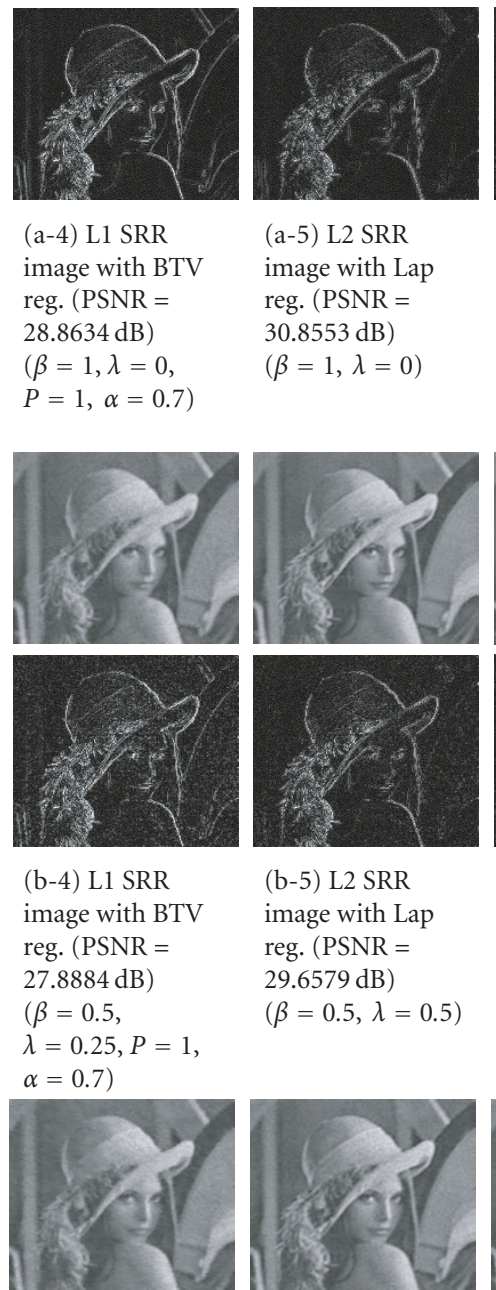

(a-5) L2 SRR image with Lap reg. $(\mathrm{PSNR}=$ $30.8553 \mathrm{~dB})$ $(\beta=1, \lambda=0)$
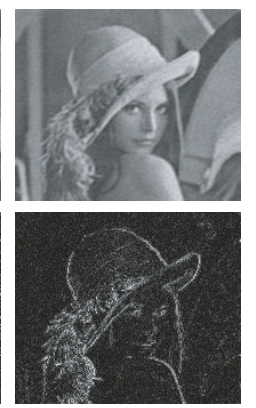

(b-5) L2 SRR image with Lap reg. $(\mathrm{PSNR}=$ $29.6579 \mathrm{~dB})$ $(\beta=0.5, \lambda=0.5)$
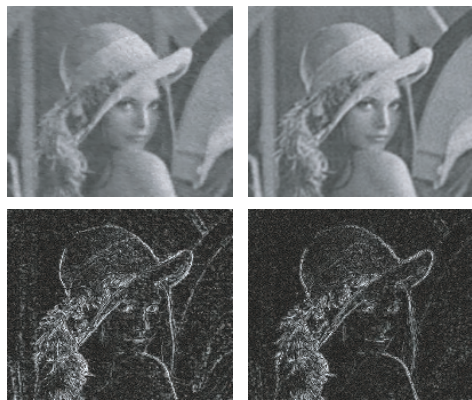

(c-4) L1 SRR image with BTV reg. $(\mathrm{PSNR}=$ $27.3968 \mathrm{~dB})$

$(\beta=0.5$,

$\lambda=0.75, P=1$, $\alpha=0.7$ )

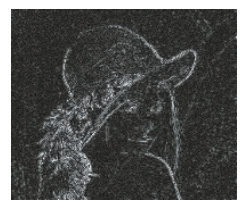
image with Lap reg. $(\mathrm{PSNR}=$ $29.1611 \mathrm{~dB})$ $(\beta=0.5, \lambda=1)$
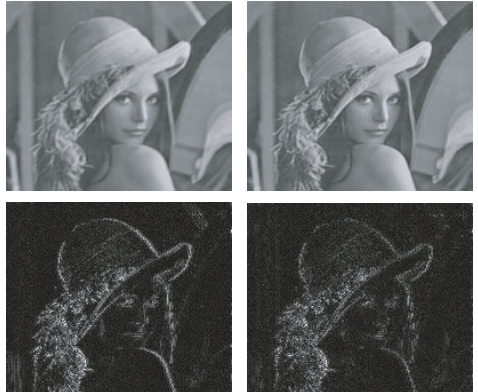

(a-6) L2 SRR image with BTV reg. $(\mathrm{PSNR}=$ $30.8553 \mathrm{~dB})$

$(\beta=1, \lambda=0$, $P=1, \alpha=0.7)$
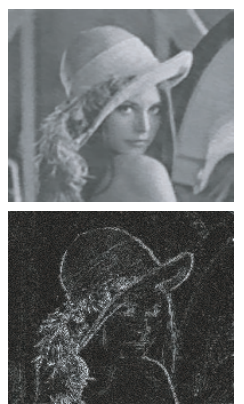

(b-6) L2 SRR image with BTV reg. $(\mathrm{PSNR}=$ $29.58 \mathrm{~dB})(\beta=$ $0.5, \lambda=0.25$, $P=1, \alpha=0.7)$

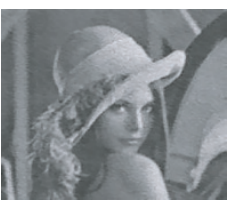

(c-5) L2 SRR

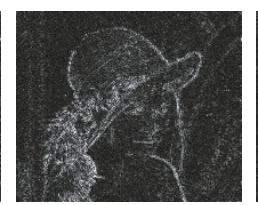

(c-6) L2 SRR image with BTV reg. $(\mathrm{PSNR}=$ $29.0775 \mathrm{~dB})$ $(\beta=0.5$, $\lambda=0.25$, $P=1, \alpha=0.7)$

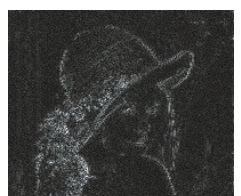

(a-7) Lor. SRR image with Lap reg. $(\mathrm{PSNR}=$ $31.9565 \mathrm{~dB})$

$(\beta=0.25, \lambda=0$, $T=3$ )

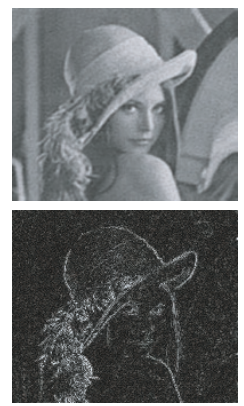

(b-7) Lor. SRR image with Lap reg. $(\mathrm{PSNR}=$ $29.7359 \mathrm{~dB})$ $(\beta=1, \lambda=0.5$, $T=15)$
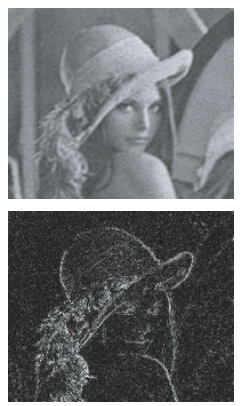

(c-7) Lor. SRR image with Lap reg. $(\mathrm{PSNR}=$ $29.1927 \mathrm{~dB})$ $(\beta=0.5, \lambda=1$,

$T=19)$

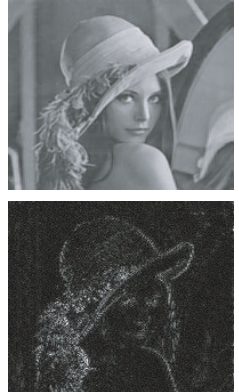

(a-8) Lor. SRR image with Lor-Lap reg. $(\mathrm{PSNR}=$

$31.9565 \mathrm{~dB})$ $(\beta=0.25, \lambda=0$, $T=3, T_{g}=1$ )

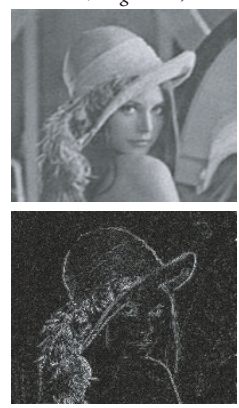

(b-8) Lor. SRR image with Lor--Lap reg. $(\mathrm{PSNR}=$ $29.7712 \mathrm{~dB})$ $(\beta=0.5, \lambda=0.5$, $\left.T=19, T_{g}=5\right)$
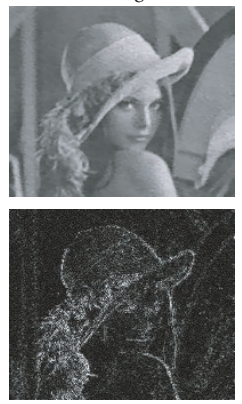

(c-8) Lor. SRR image with Lor.-Lap reg. $(\mathrm{PSNR}=$ $29.2183 \mathrm{~dB}$ ) $(\beta=0.5, \lambda=0.75$, $T=19, T_{g}=9$ )

FIgURE 4: The experimental results of proposed method. 


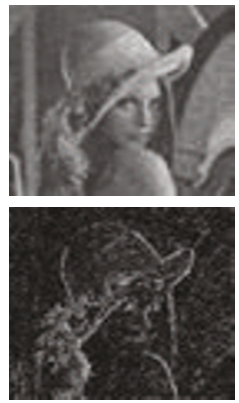

(d-2) Corrupted LR image (AWGN:SNR = $20 \mathrm{~dB})(\mathrm{PSNR}=$ $26.2188 \mathrm{~dB})$
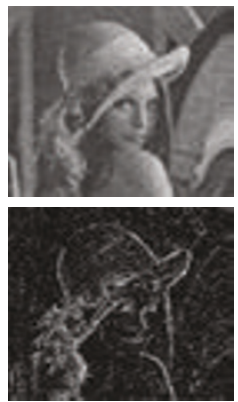

(d-3) L1 SRR image with Lap reg. $(\mathrm{PSNR}=$ $26.7854 \mathrm{~dB})$ $(\beta=0.5, \lambda=1.0)$
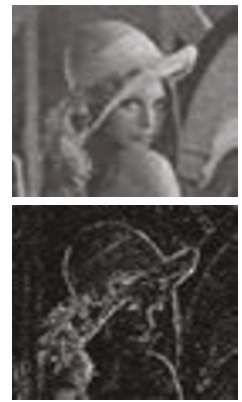

(d-4) L1 SRR image with BTV reg. $(\mathrm{PSNR}=$ $26.7197 \mathrm{~dB}$ )

$(\beta=0.5$,

$\lambda=0.8, P=1$, $\alpha=0.7$ )
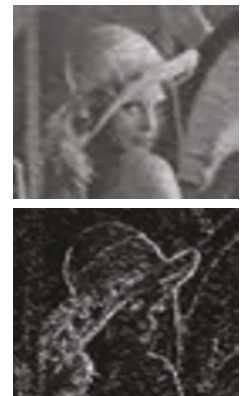

(e-4) L1 SRR image with BTV reg. $(\mathrm{PSNR}=$ $26.0066 \mathrm{~dB})$

( $\beta=0.5$,

$\lambda=0.75$,

$P=1, \alpha=0.7)$

$26.0348 \mathrm{~dB})$

$(\beta=0.5, \lambda=1)$
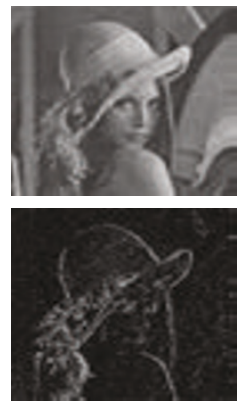

(d-5) L2 SRR image with Lap reg. $(\mathrm{PSNR}=$ $28.6024 \mathrm{~dB})$ $(\beta=0.5, \lambda=1)$
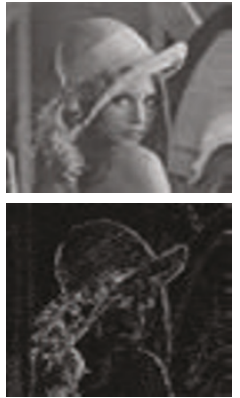

(d-6) L2 SRR image with BTV reg. (PSNR $=$ $28.5195 \mathrm{~dB})$

$(\beta=0.5$,

$\lambda=0.5, P=1$, $\alpha=0.7$ )
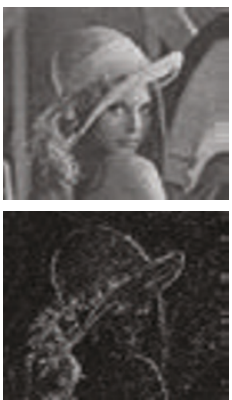

(e-5) L2 SRR image with Lap reg. $(\mathrm{PSNR}=$ $27.8153 \mathrm{~dB})$ $(\beta=0.5, \lambda=1)$
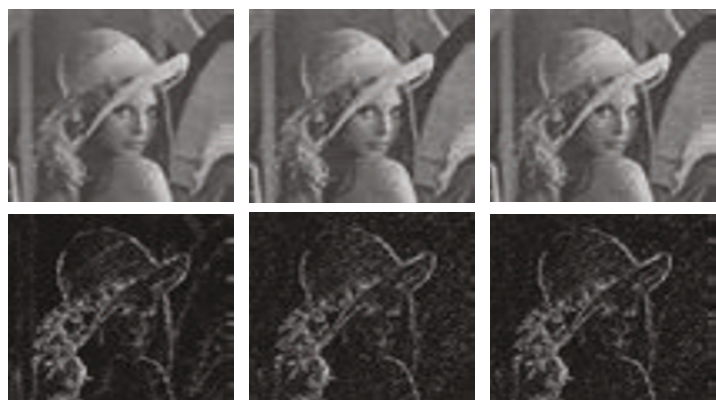

(e-6) L2 SRR image with BTV reg. $(\mathrm{PSNR}=$ $27.964 \mathrm{~dB})$ ( $\beta=0.5$, $\lambda=0.75$, $P=1, \alpha=0.7)$

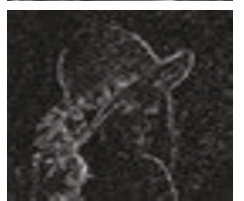

(e-7) Lor. SRR image with Lap reg. $(\mathrm{PSNR}=$ $27.7621 \mathrm{~dB})$ $(\beta=0.5, \lambda=1$,

$T=15)$

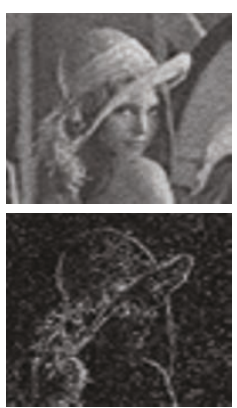

(f-7) Lor. SRR image with Lap reg. $(\mathrm{PSNR}=$ $26.7566 \mathrm{~dB})$ $(\beta=0.5, \lambda=1$, $T=9)$

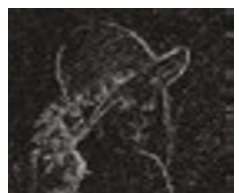

(e-8) Lor. SRR image with Lor.-Lap reg. $(\mathrm{PSNR}=$ $27.9152 \mathrm{~dB})$ $(\beta=0.5, \lambda=1$, $\left.T=15, T_{g}=15\right)$

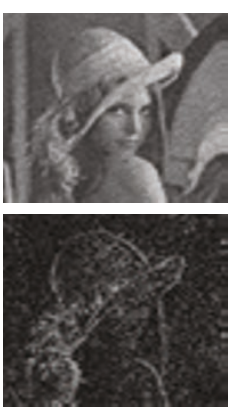

(f-5) L2 SRR image with Lap reg. (PSNR = $26.6406 \mathrm{~dB})$ $(\beta=0.5, \lambda=1)$

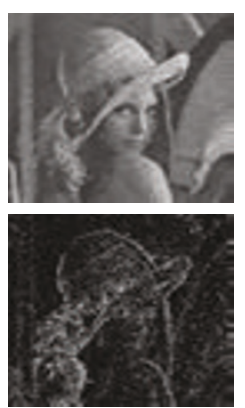

(f-6) L2 SRR image with BTV reg. (PSNR $26.7713 \mathrm{~dB})$ $(\beta=0.5, \lambda=0.7$, $P=1, \alpha=0.7)$
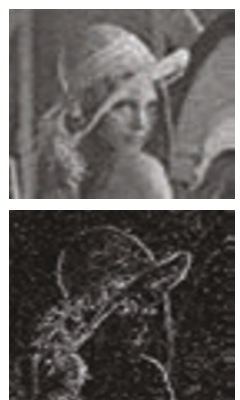

(f-8) Lor. SRR image with Lor.-Lap reg. $(\mathrm{PSNR}=$ $26.7947 \mathrm{~dB})$ ( $\beta=0.5, \lambda=1$, $T=5, T_{g}=9$ )
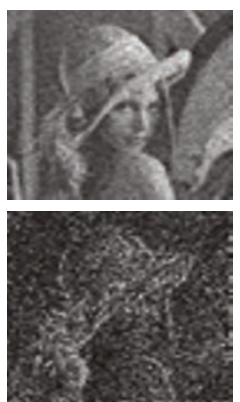

(f-2) Corrupted LR image

(AWGN:SNR = $15 \mathrm{~dB})(\mathrm{PSNR}=$ $23.3549 \mathrm{~dB}$ )

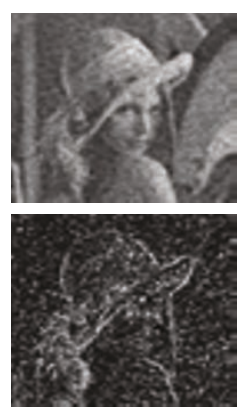

(f-3) L1 SRR image with Lap reg. $(\mathrm{PSNR}=$ $(\beta=0.5, \lambda=1)$
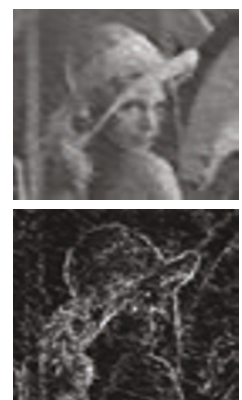

(f-4) L1 SRR with BT $25.2642 \mathrm{~dB})$ $(\beta=0.5$, $\lambda=0.8$, $P=1, \alpha=0.7$ ) 

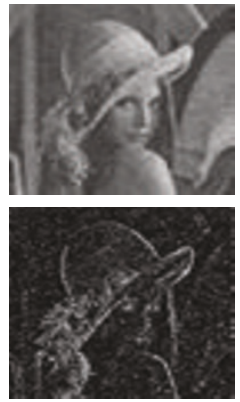

(g-2) Corrupted LR image (Poisson) (PSNR $=26.5116 \mathrm{~dB}$ )
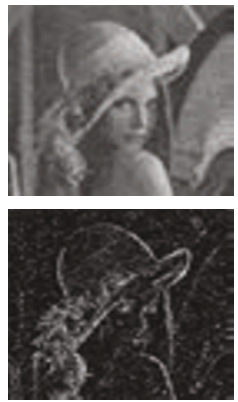

(g-3) L1 SRR image with Lap reg. $(\mathrm{PSNR}=$ $26.9604 \mathrm{~dB})$ $(\beta=0.5, \lambda=1)$
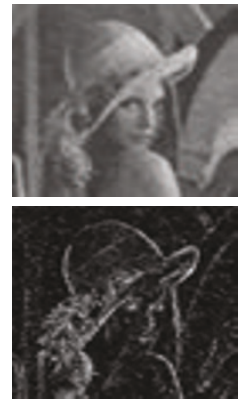

(g-4) L1 SRR image with BTV reg. $(\mathrm{PSNR}=$ $26.8759 \mathrm{~dB})$ $(\beta=0.5, \lambda=0.8$, $P=1, \alpha=0.7)$
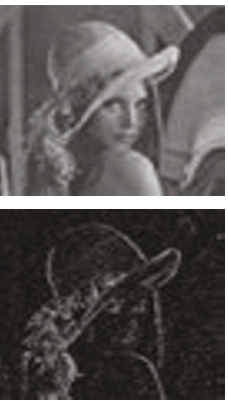

(g-5) L2 SRR image with Lap reg. $(\mathrm{PSNR}=$ $28.719 \mathrm{~dB})$ $(\beta=0.5, \lambda=1)$
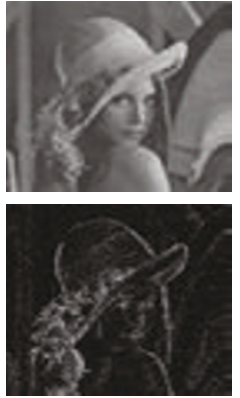

(g-6) L2 SRR image with BTV reg. $(\mathrm{PSNR}=$ $28.6848 \mathrm{~dB})$ $(\beta=0.5, \lambda=0.5$, $P=1, \alpha=0.7$ )
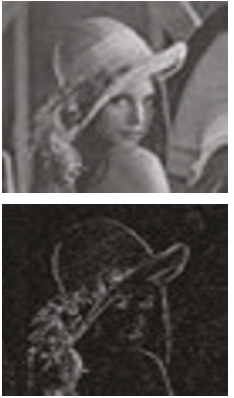

(g-7) Lor. SRR image with Lap reg. $(\mathrm{PSNR}=$ $28.6735 \mathrm{~dB})$ $(\beta=0.5, \lambda=1$, $T=19$ )
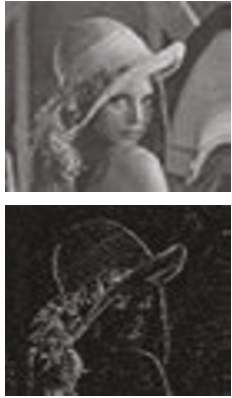

(g-8) Lor. SRR image with Lor--Lap reg. (PSNR = $28.7471 \mathrm{~dB})$ $(\beta=0.5, \lambda=1$, $\left.T=19, T_{g}=5\right)$

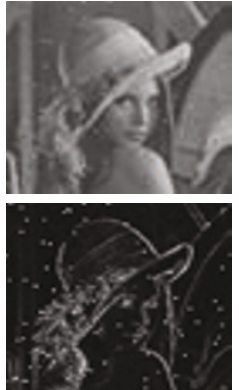

(h-2) Corrupted LR image (S\&P: $D=0.005)$ $(\mathrm{PSNR}=$ $26.8577 \mathrm{~dB}$ )
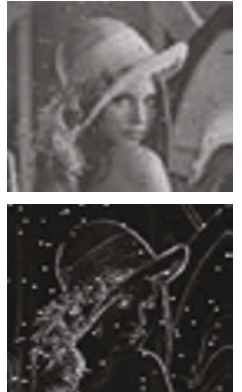

(h-3) L1 SRR image with Lap reg. $(\mathrm{PSNR}=$ $27.1149 \mathrm{~dB})$ $(\beta=0.5, \lambda=1)$
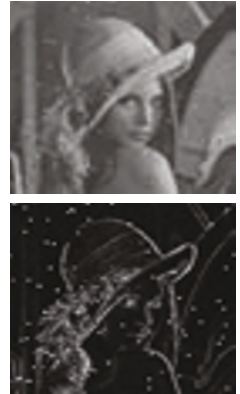

(h-4) L1 SRR image with BTV reg. $(\mathrm{PSNR}=$ $26.8577 \mathrm{~dB})$ $(\beta=1, \lambda=0.5$, $P=1, \alpha=0.7$ )
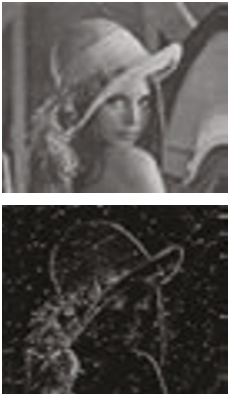

(h-5) L2 SRR image with Lap reg. $(\mathrm{PSNR}=$ $28.8495 \mathrm{~dB})$ $(\beta=0.5, \lambda=1)$
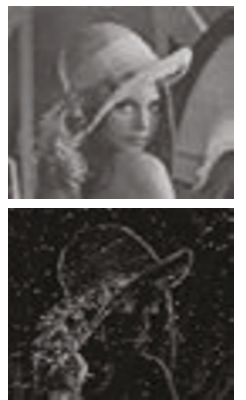

(h-6) L2 SRR image with BTV reg. $(\mathrm{PSNR}=$ $28.1438 \mathrm{~dB})$ $(\beta=0.5, \lambda=0.6$, $P=1, \alpha=0.7$ )
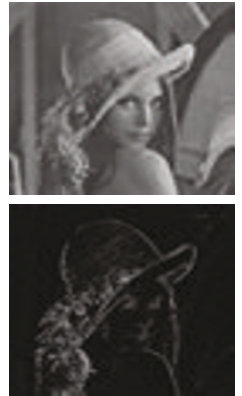

(h-7) Lor. SRR image with Lap reg. $(\mathrm{PSNR}=$ $31.1843 \mathrm{~dB})$ $(\beta=1, \lambda=0.25$, $T=9)$
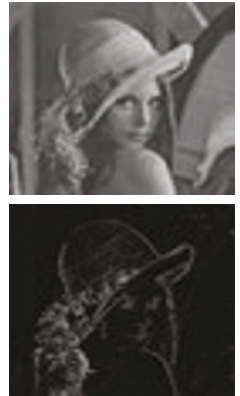

(h-8) Lor. SRR image with Lor.-Lap reg. $(\mathrm{PSNR}=$

$31.2123 \mathrm{~dB})$ $(\beta=1, \lambda=0.5$, $T=9, T_{g}=1$ )
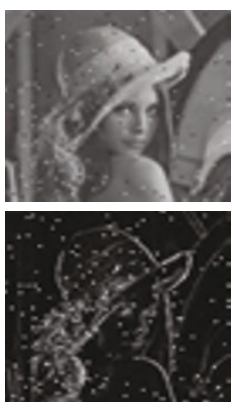

(i-2) (S\&P:

$D=0.010)$

Corrupted LR image $(\mathrm{PSNR}=$ $25.2677 \mathrm{~dB}$ )
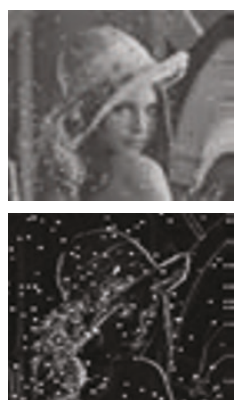

(i-3) L1 SRR image with Lap reg. $(\mathrm{PSNR}=$ $26.0569 \mathrm{~dB})$ $(\beta=1, \lambda=1)$
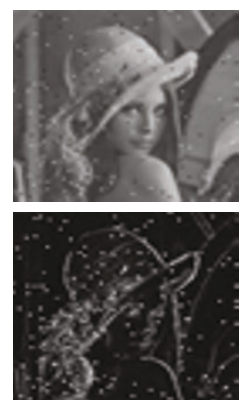

(i-4) L1 SRR image with BTV reg. $(\mathrm{PSNR}=$ $25.2677 \mathrm{~dB})$

$(\beta=1, \lambda=0.4$, $P=1, \alpha=0.7)$
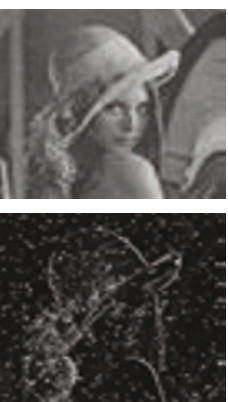

(i-5) L2 SRR image with Lap reg. $(\mathrm{PSNR}=$ $28.0346 \mathrm{~dB})$ $(\beta=0.5, \lambda=1)$

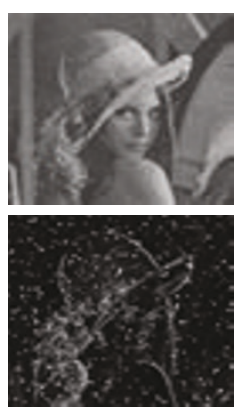

(i-6) L2 SRR image with BTV reg. $(\mathrm{PSNR}=$ $26.7979 \mathrm{~dB})$ $(\beta=0.5, \lambda=0.4$, $P=1, \alpha=0.7$ )

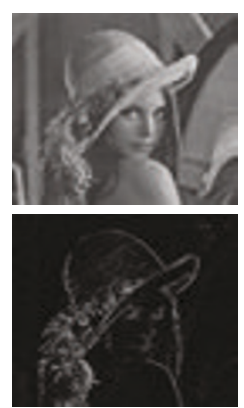

(i-7) Lor. SRR image with Lap reg. $(\mathrm{PSNR}=$ $31.0524 \mathrm{~dB})$ $(\beta=1, \lambda=0.25$, $T=19)$

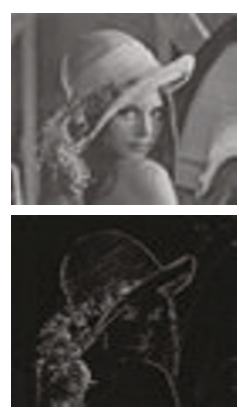

(i-8) Lor. SRR image with Lor.-Lap reg. $(\mathrm{PSNR}=$ $31.0748 \mathrm{~dB})$ $(\beta=1, \lambda=0.25$, $T=9, T_{g}=5$ ) 

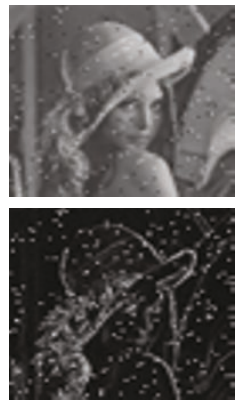

(j-2) Corrupted LR image (S\&P: $D=0.015$ ) $(\mathrm{PSNR}=$ $24.2190 \mathrm{~dB})$
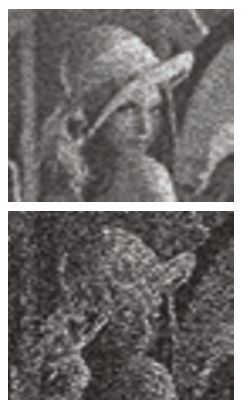

(k-2) (Speckle) Corrupted LR image $(\mathrm{PSNR}=$ $21.7994 \mathrm{~dB}$ )
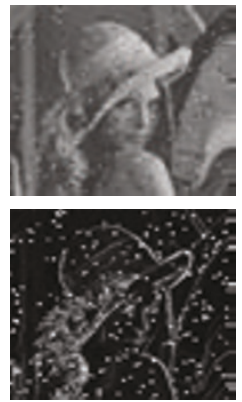

(j-3) L1 SRR image with Lap reg. $(\mathrm{PSNR}=$ $25.3534 \mathrm{~dB})$ $(\beta=1, \lambda=1)$
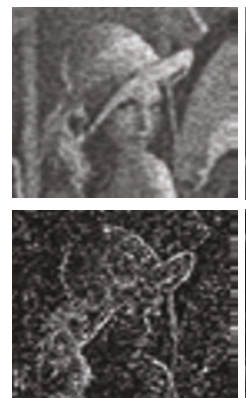

(k-3) L1 SRR image with Lap reg. $(\mathrm{PSNR}=$ $24.4215 \mathrm{~dB})$ $(\beta=0.5, \lambda=1)$
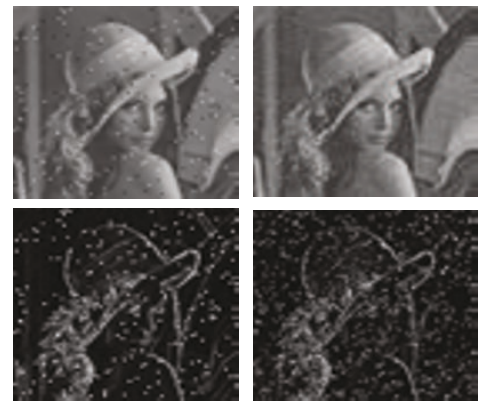

(j-4) L1 SRR image with BTV reg. $(\mathrm{PSNR}=$ $24.2202 \mathrm{~dB})$ $(\beta=0.5, \lambda=0.3$, $P=1, \alpha=0.7$ )
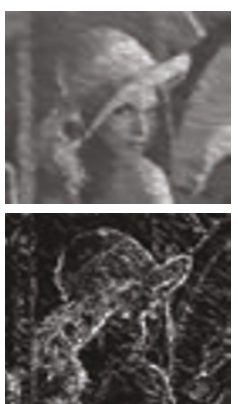

(k-4) L1 SRR image with BTV reg. $(\mathrm{PSNR}=$ $24.5102 \mathrm{~dB})$ $(\beta=0.5, \lambda=0.5$, $P=1, \alpha=0.7)$

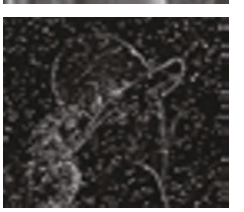

\section{(j-5) L2 SRR} image with Lap reg. $(\mathrm{PSNR}=$ $27.3188 \mathrm{~dB})$

$(\beta=0.5, \lambda=1)$
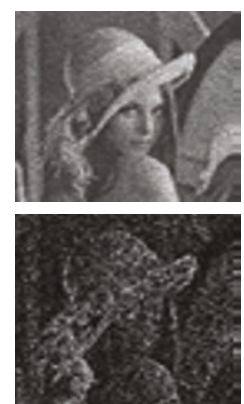

(k-5) L2 SRR image with Lap reg. $(\mathrm{PSNR}=$ $25.3165 \mathrm{~dB})$ $(\beta=0.5, \lambda=1)$
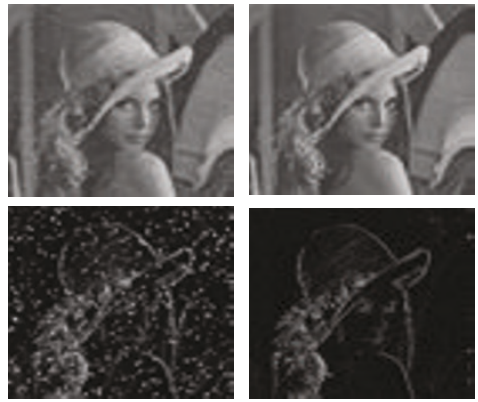

(j-6) L2 SRR image with BTV reg. $(\mathrm{PSNR}=$ $25.8242 \mathrm{~dB})$ $(\beta=0.5, \lambda=0.4$, $P=1, \alpha=0.7)$
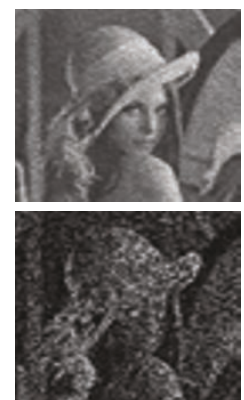

(k-6) L2 SRR image with BTV reg. $(\mathrm{PSNR}=$ $23.958 \mathrm{~dB})$ $(\beta=0.5, \lambda=0.4$, $P=1, \alpha=0.7)$

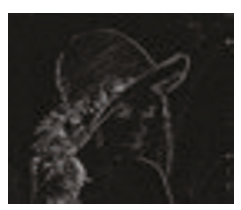

(j-7) Lor. SRR image with Lap reg. $(\mathrm{PSNR}=$ $30.0229 \mathrm{~dB})$ $(\beta=1, \lambda=0.25$, $T=19)$
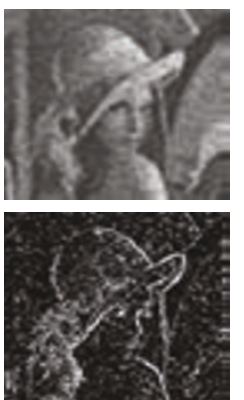

(k-7) Lor. SRR image with Lap reg. $(\mathrm{PSNR}=$ $25.3136 \mathrm{~dB})$ $(\beta=0.5, \lambda=1$, $T=1$ )

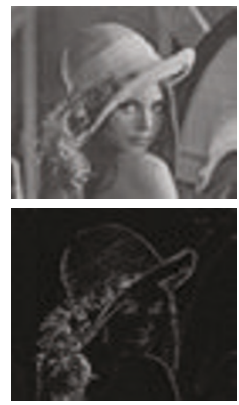

(j-8) Lor. SRR image with Lor.-Lap reg. $(\mathrm{PSNR}=$ $31.0627 \mathrm{~dB})$ $(\beta=1, \lambda=0.25$, $\left.T=9, T_{g}=5\right)$

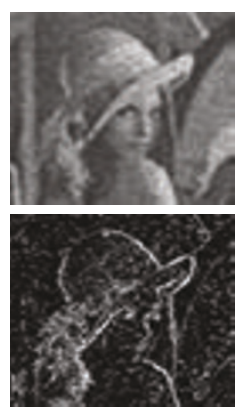

(k-8) Lor. SRR image with

Lor.-Lap reg.

$(\mathrm{PSNR}=$

$25.609 \mathrm{~dB})$

$(\beta=0.5, \lambda=1$, $\left.T=1, T_{g}=5\right)$

Figure 4: continued.

regularization, L2 estimator with BTV regularization, Lorentzian estimator with Laplacian regularization and Lorentzian estimator with Lorentzian-Laplacian regularization are shown in Figures 4(a-3)-4(a-8), respectively.

\section{Additive white Gaussian noise}

This experiment is 5 AWGN cases at $\mathrm{SNR}=25,22.5,20$, 17.5 , and $15 \mathrm{~dB}$, respectively, and the original HR images are shown in Figures 4(b-1) $-4(\mathrm{f}-1)$, respectively. The corrupted images at $\mathrm{SNR}=25,22.5,20,17.5$, and $15 \mathrm{~dB}$ are shown in Figures $4(\mathrm{~b}-2)-4(\mathrm{f}-2)$, respectively.

At $\mathrm{SNR}=25,22.5,20,17.5$, and $15 \mathrm{~dB}$, respectively, the results of L1 estimator with Laplacian regularization, L1 estimator with BTV regularization, L2 estimator with Laplacian regularization, L2 estimator with BTV regularization, Lorentzian estimator with Laplacian regularization and Lorentzian estimator with Lorentzian-Laplacian regularization estimation are shown in Figures 4(b-3)-4(b-8), 4(c-3)$4(\mathrm{c}-8), 4(\mathrm{~d}-3)-4(\mathrm{~d}-8), 4(\mathrm{e}-3)-4(\mathrm{e}-8)$, and $4(\mathrm{f}-3)-4(\mathrm{f}-8)$, respectively. The Lorentzian estimator result (with LorentzianLaplacian and Laplacian regularization) gives the best per- formance than L2 estimator result (with Laplacian and BTV regularization) and L1 estimator result (with Laplacian and BTV regularizations).

\section{Poisson noise}

The original HR image is shown in Figure 4(g-1) and one of corrupted LR images is shown in Figure $4(\mathrm{~g}-2)$. The Lorentzian estimator result with Lorentzian-Laplacian regularization gives the highest PSNR than the Lorentzian estimator result with Laplacian regularization, L2 estimator result with Laplacian and BTV regularization and L1 estimator result with Laplacian and BTV regularization. The result of implementing the super-resolution method using L1 estimator with Laplacian regularization, L1 estimator with BTV regularization, L2 estimator with Laplacian regularization, L2 estimator with BTV regularization, Lorentzian estimator with Laplacian regularization and Lorentzian estimator with Lorentzian-Laplacian regularization are shown in Figures $4(\mathrm{~g}-3)-4(\mathrm{~g}-8)$, respectively. 


\section{Salt and pepper noise}

This experiment is 3 salt and pepper noise cases at $D=0.005$, $D=0.01$ and $D=0.015$, respectively, and the original HR images are shown in Figures $4(\mathrm{~h}-1)-4(\mathrm{j}-1)$ respectively. The corrupted images at $D=0.005, D=0.010$ and $D=0.015$ are shown in Figures 4(h-2), 4(i-2), and 4(j-2), respectively. The Lorentzian estimator results (with Lorentzian-Laplacian and Laplacian regularization) give dramatically higher PSNR than L1 estimator result (with Laplacian and BTV regularization results) and L2 estimator results (with Laplacian and BTV regularizations results).

At $D=0.005, D=0.01$, and $D=0.015$, the results of L1 estimator with Laplacian regularization, L1 estimator with BTV regularization, L2 estimator with Laplacian regularization, L2 estimator with BTV regularization, Lorentzian estimator with Laplacian regularization and Lorentzian estimator with Lorentzian-Laplacian regularization estimation are shown in Figures 4(h-3)-4(h-8), 4(i-3)-4(i-8), and 4(j4) $-4(\mathrm{j}-8)$, respectively.

\section{Speckle noise}

The last experiment is speckle noise cases and the original HR images are shown in Figure $4(\mathrm{k}-1)$. The corrupted image is shown in Figure 4(k-2). The Lorentzian estimator result (with Lorentzian-Laplacian and Laplacian regularization) gives the best performance than L2 estimator result (with Laplacian and BTV regularizations) and L1 estimator result (with Laplacian and BTV regularization). The result of L1 estimator with Laplacian regularization, L1 estimator with BTV regularization, L2 estimator with Laplacian regularization, L2 estimator with BTV regularization, Lorentzian estimator with Laplacian regularization and Lorentzian estimator with Lorentzian-Laplacian regularization are shown in Figures 4(k-3)-4(k-8).

\section{CONCLUSION}

In this paper, we propose an alternate approach using a novel robust estimation norm function (Lorentzian norm function) for SRR framework with Tikhonov and LorentzianTikhonov regularizations and the proposed robust SRR can be effectively applied on the images that is corrupted by various noise models. Experimental results conducted clearly that the proposed robust algorithm can well apply on the several noise models such as noiseless, AWGN, Poisson noise, and salt and pepper noise, and speckle noise and the proposed algorithm can obviously improve the result in using both subjective and objective measurements.

\section{ACKNOWLEDGMENTS}

The authors would like to express the grateful thanks to grant from government research and development in cooperative project between Department of Electrical Engineering and Private Sector Research for supporting this work and development under Chulalongkorn University.

\section{REFERENCES}

[1] C. A. Segall, R. Molina, and A. K. Katsaggelos, "Highresolution images from low-resolution compressed video," IEEE Signal Processing Magazine, vol. 20, no. 3, pp. 37-48, 2003.

[2] D. Kundur and D. Hatzinakos, "Blind image deconvolution," IEEE Signal Processing Magazine, vol. 13, no. 3, pp. 43-64, 1996.

[3] D. Rajan, S. Chaudhuri, and M. V. Joshi, "Multi-objective super resolution: concepts and examples," IEEE Signal Processing Magazine, vol. 20, no. 3, pp. 49-61, 2003.

[4] G. Demoment, "Image reconstruction and restoration: overview of common estimation structures and problems," IEEE Transactions on Acoustics, Speech, and Signal Processing, vol. 37, no. 12, pp. 2024-2036, 1989.

[5] M. K. Ng and N. K. Bose, "Mathematical analysis of superresolution methodology," IEEE Signal Processing Magazine, vol. 20, no. 3, pp. 62-74, 2003.

[6] M. G. Kang and S. Chaudhuri, "Super-resolution image reconstruction," IEEE Signal Processing Magazine, vol. 20, no. 3, pp. 19-20, 2003.

[7] S. Chaudhuri and D. R. Taur, "High-resolution slow-motion sequencing," IEEE Signal Processing Magazine, vol. 22, no. 2, pp. 16-24, 2005.

[8] S. C. Park, M. K. Park, and M. G. Kang, "Super-resolution image reconstruction: a technical overview," IEEE Signal Processing Magazine, vol. 20, no. 3, pp. 21-36, 2003.

[9] M. R. Banham and A. K. Katsaggelos, "Digital image restoration," IEEE Signal Processing Magazine, vol. 14, no. 2, pp. 2441, 1997.

[10] T. S. Huang and R. Y. Tsan, "Multiple frame image restoration and registration," in Advances in Computer Vision and Image Processing, T. S. Huang, Ed., vol. 1, pp. 317-339, JAI Press, Greenwich, Conn, USA, 1984.

[11] S. P. Kim, N. K. Bose, and H. M. Valenzuela, "Recursive reconstruction of high resolution image from noisy undersampled multiframes," IEEE Transactions on Acoustics, Speech, and Signal Processing, vol. 38, no. 6, pp. 1013-1027, 1990.

[12] S. P. Kim and W.-Y. Su, "Recursive high-resolution reconstruction of blurred multiframe images," IEEE Transactions on Image Processing, vol. 2, no. 4, pp. 534-539, 1993.

[13] M. K. Ng and N. K. Bose, "Analysis of displacement errors in high-resolution image reconstruction with multisensors," IEEE Transactions on Circuits and Systems I: Fundamental Theory and Applications, vol. 49, no. 6, pp. 806-813, 2002.

[14] N. K. Bose, M. K. Ng, and A. C. Yau, "A fast algorithm for image super-resolution from blurred observations," EURASIP Journal on Applied Signal Processing, vol. 2006, Article ID 35726, 14 pages, 2006.

[15] A. J. Patti and Y. Altunbasak, "Artifact reduction for set theoretic super resolution image reconstruction with edge adaptive constraints and higher-order interpolants," IEEE Transactions on Image Processing, vol. 10, no. 1, pp. 179-186, 2001.

[16] Y. Altunbasak, A. J. Patti, and R. M. Mersereau, "Superresolution still and video reconstruction from MPEG-coded video," IEEE Transactions on Circuits and Systems for Video Technology, vol. 12, no. 4, pp. 217-226, 2002.

[17] B. K. Gunturk, Y. Altunbasak, and R. M. Mersereau, "Super-resolution reconstruction of compressed video using transform-domain statistics," IEEE Transactions on Image Processing, vol. 13, no. 1, pp. 33-43, 2004. 
[18] H. Hasegawa, T. Ono, I. Yamada, and K. Sakaniwa, "An iterative MPEG super-resolution with an outer approximation of framewise quantization constraint," IEICE Transactions on Fundamentals of Electronics, Communications and Computer Sciences, vol. E88-A, no. 9, pp. 2427-2434, 2005.

[19] I. M. Elfadel and R. W. Picard, "Miscibility matrices explain the behavior of grayscale textures generated by Gibbs random fields," in Intelligent Robots and Computer Vision IX: Algorithms and Techniques, vol. 1381 of Proceedings of SPIE, pp. 524-535, Boston, Mass, USA, November 1991.

[20] I. M. Elfadel and R. W. Picard, "Gibbs random fields, cooccurrences, and texture modeling," Perceptual Computing Group Tech. Rep. \#204, pp. 1-34, Media Laboratory, MIT, Cambridge, Mass, USA, January 1993.

[21] I. M. Elfadel and R. W. Picard, "Gibbs random fields, cooccurences, and texture modeling," IEEE Transactions on Pattern Analysis and Machine Intelligence, vol. 16, no. 1, pp. 24-37, 1994.

[22] I. M. Elfadel and R. W. Picard, "On the structure of aura and co-occurrence matrices for the Gibbs texture model," Perceptual Computing Group Tech. Rep. \#160, pp. 1-24, Media Laboratory, MIT, Cambridge, Mass, USA, June 1994.

[23] R. W. Picard, "Gibbs random fields: temperature and parameter analysis," in Proceedings of IEEE International Conference on Acoustics, Speech, and Signal Processing (ICASSP '92), vol. 3 , pp. 45-48, San Francisco, Calif, USA, March 1992.

[24] K. Popat and R. W. Picard, "Cluster-based probability model applied to image restoration and compression," in Proceedings of IEEE International Conference on Acoustics, Speech, and Signal Processing (ICASSP '94), vol. 5, pp. 381-384, Adelaide, SA, Australia, April 1994.

[25] R. W. Picard, I. M. Elfadel, and A. P. Pentland, "Markov/Gibbs texture modeling: aura matrices and temperature effects," in Proceedings of IEEE Computer Society Conference on Computer Vision and Pattern Recognition (CVPR'91), pp. 371-377, Maui, Hawaii, USA, June 1991.

[26] R. W. Picard, "Gibbs random fields: temperature and parameter analysis," in IEEE International Conference on Acoustics, Speech, and Signal Processing (ICASSP '92), vol. 3, pp. 45-48, San Francisco, Calif, USA, March 1992.

[27] C. Bouman and K. Sauer, "A generalized Gaussian image model for edge-preserving MAP estimation," IEEE Transactions on Image Processing, vol. 2, no. 3, pp. 296-310, 1993.

[28] R. R. Schultz and R. L. Stevenson, "A Bayesian approach to image expansion for improved definition," IEEE Transactions on Image Processing, vol. 3, no. 3, pp. 233-242, 1994.

[29] R. R. Schultz and R. L. Stevenson, "Extraction of highresolution frames from video sequences," IEEE Transactions on Image Processing, vol. 5, no. 6, pp. 996-1011, 1996.

[30] R. Pan and S. J. Reeves, "Efficient Huber-Markov edgepreserving image restoration," IEEE Transactions on Image Processing, vol. 15, no. 12, pp. 3728-3735, 2006.

[31] D. Kundur and D. Hatzinakos, "Blind image deconvolution revisited," IEEE Signal Processing Magazine, vol. 13, no. 6, pp. 61-63, 1996.

[32] A. M. Thompson, J. C. Brown, J. W. Kay, and D. M. Titterington, "A study of methods of choosing the smoothing parameter in image restoration by regularization," IEEE Transactions on Pattern Analysis and Machine Intelligence, vol. 13, no. 4, pp. 326-339, 1991.

[33] V. Z. Mesarovic, N. P. Galatsanos, and A. K. Katsaggelos, "Regularized constrained total least squares image restoration," IEEE Transactions on Image Processing, vol. 4, no. 8, pp. 10961108, 1995.
[34] D. Geman and C. Yang, "Nonlinear image recovery with halfquadratic regularization," IEEE Transactions on Image Processing, vol. 4, no. 7, pp. 932-946, 1995.

[35] M. G. Kang and A. K. Katsaggelos, "General choice of the regularization functional in regularized image restoration," IEEE Transactions on Image Processing, vol. 4, no. 5, pp. 594-602, 1995.

[36] M. G. Kang and A. K. Katsaggelos, "Simultaneous multichannel image restoration and estimation of the regularization parameters," IEEE Transactions on Image Processing, vol. 6, no. 5, pp. 774-778, 1997.

[37] R. Molina, A. K. Katsaggelos, and J. Mateos, "Bayesian and regularization methods for hyperparameter estimation in image restoration," IEEE Transactions on Image Processing, vol. 8, no. 2, pp. 231-246, 1999.

[38] R. Molina, M. Vega, J. Abad, and A. K. Katsaggelos, "Parameter estimation in Bayesian high-resolution image reconstruction with multisensors," IEEE Transactions on Image Processing, vol. 12, no. 12, pp. 1655-1667, 2003.

[39] D. Rajan and S. Chaudhuri, "Simultaneous estimation of super-resolved scene and depth map from low resolution defocused observations," IEEE Transactions on Pattern Analysis and Machine Intelligence, vol. 25, no. 9, pp. 1102-1117, 2003.

[40] H. He and L. P. Kondi, "Resolution enhancement of video sequences with adaptively weighted low-resolution images and simultaneous estimation of the regularization parameter," in Proceedings of IEEE International Conference on Acoustics, Speech, and Signal Processing (ICASSP'04), vol. 3, pp. 213-216, Montreal, Que, Canada, May 2004.

[41] H. He and L. P. Kondi, "Resolution enhancement of video sequences with simultaneous estimation of the regularization parameter," Journal of Electronic Imaging, vol. 13, no. 3, pp. 586-596, 2004.

[42] H. He and L. P. Kondi, "A regularization framework for joint blur estimation and super-resolution of video sequences," in Proceedings of International Conference on Image Processing (ICIP '05), vol. 3, pp. 329-332, Genova, Italy, September 2005.

[43] H. He and L. P. Kondi, "An image super-resolution algorithm for different error levels per frame," IEEE Transactions on Image Processing, vol. 15, no. 3, pp. 592-603, 2006.

[44] M. Vega, R. Molina, and A. K. Katsaggelos, "A Bayesian super-resolution approach to demosaicing of blurred images," EURASIP Journal on Applied Signal Processing, vol. 2006, Article ID 25072, 12 pages, 2006.

[45] M. Elad and A. Feuer, "Restoration of a single superresolution image from several blurred, noisy, and undersampled measured images," IEEE Transactions on Image Processing, vol. 6, no. 12, pp. 1646-1658, 1997.

[46] M. Elad and A. Feuer, "Superresolution restoration of an image sequence: adaptive filtering approach," IEEE Transactions on Image Processing, vol. 8, no. 3, pp. 387-395, 1999.

[47] M. Elad and A. Feuer, "Super-resolution restoration of continuous image sequence-adaptive filtering approach," Tech. Rep. \#942, pp. 1-12, The Technion, the Electrical Engineering Faculty, Israel Institute of Technology, Haifa, Israel, 1994.

[48] M. Elad and A. Feuer, "Super-resolution reconstruction of image sequences," IEEE Transactions on Pattern Analysis and Machine Intelligence, vol. 21, no. 9, pp. 817-834, 1999.

[49] M. Elad and Y. Hel-Or, "A fast super-resolution reconstruction algorithm for pure translational motion and common spaceinvariant blur," IEEE Transactions on Image Processing, vol. 10, no. 8, pp. 1187-1193, 2001.

[50] N. Nguyen, P. Milanfar, and G. Golub, "A computationally efficient superresolution image reconstruction algorithm," IEEE 
Transactions on Image Processing, vol. 10, no. 4, pp. 573-583, 2001.

[51] M. Elad, "On the origin of the bilateral filter and ways to improve it," IEEE Transactions on Image Processing, vol. 11, no. 10, pp. 1141-1151, 2002.

[52] S. Farsiu, D. Robinson, M. Elad, and P. Milanfar, "Advances and challenges in super-resolution," International Journal of Imaging Systems and Technology, vol. 14, no. 2, pp. 47-57, 2004.

[53] S. Farsiu, D. Robinson, M. Elad, and P. Milanfar, "Fast and robust multiframe super resolution," IEEE Transactions on Image Processing, vol. 13, no. 10, pp. 1327-1344, 2004.

[54] S. Farsiu, M. Elad, and P. Milanfar, "Multiframe demosaicing and super-resolution of color images," IEEE Transactions on Image Processing, vol. 15, no. 1, pp. 141-159, 2006.

[55] S. Farsiu, M. Elad, and P. Milanfar, "Video-to-video dynamic super-resolution for grayscale and color sequences," EURASIP Journal on Applied Signal Processing, vol. 2006, Article ID 61859, 15 pages, 2006.

[56] V. Patanavijit and S. Jitapunkul, "An iterative super-resolution reconstruction of image sequences using a Bayesian approach and affine block-based registration," in Proceedings of the 14th European Signal Processing Conference (EUSIPCO '06), Florence, Italy, September 2006.

[57] G. Rochefort, F. Champagnat, G. Le Besnerais, and J.-F. Giovannelli, "An improved observation model for superresolution under affine motion," IEEE Transactions on Image Processing, vol. 15, no. 11, pp. 3325-3337, 2006.

[58] S. Baker and T. Kanade, "Limits on super-resolution and how to break them," IEEE Transactions on Pattern Analysis and Machine Intelligence, vol. 24, no. 9, pp. 1167-1183, 2002.

[59] J. Sun, N.-N. Zheng, H. Tao, and H.-Y. Shum, "Image hallucination with primal sketch priors," in Proceedings of the IEEE Computer Society Conference on Computer Vision and Pattern Recognition (CVPR '03), vol. 2, pp. 729-736, Madison, Wis, USA, June 2003.

[60] P. Vandewalle, S. Süsstrunk, and M. Vetterli, "Double resolution from a set of aliased images," in Sensors and Camera Systems for Scientific, Industrial, and Digital Photography Applications V, vol. 5301 of Proceedings of SPIE, pp. 374-382, San Jose, Calif, USA, January 2004.

[61] P. Vandewalle, S. Süsstrunk, and M. Vetterli, "A frequency domain approach to super-resolution imaging from aliased low resolution images," Technical Journal, pp. 1-21, Department of Electrical Engineering and Computer Science, University of California, Berkeley, Calif, USA, May 2004.

[62] P. Vandewalle, L. Sbaiz, M. Vetterli, and S. Süsstrunk, "Superresolution from highly undersampled images," in Proceedings of International Conference on Image Processing (ICIP '05), vol. 1, pp. 889-892, Genova, Italy, September 2005.

[63] P. Vandewalle, S. Süsstrunk, and M. Vetterll, "A frequency domain approach to registration of aliased images with application to super-resolution," EURASIP Journal on Applied Signal Processing, vol. 2006, Article ID 71459, 14 pages, 2006.

[64] M. Trimeche, R. C. Bilcu, and J. Yrjänäinen, "Adaptive outlier rejection in image super-resolution," EURASIP Journal on Applied Signal Processing, vol. 2006, Article ID 38052, 12 pages, 2006.

[65] M. J. Black and A. Rangarajan, "On the unification of line processes, outlier rejection, and robust statistics with applications in early vision," International Journal of Computer Vision, vol. 19, no. 1, pp. 57-91, 1996.

[66] M. J. Black, G. Sapiro, D. H. Marimont, and D. Heeger, "Robust anisotropic diffusion: connections between robust statis- tics, line processing, and anisotropic diffusion," in Proceedings of the 1st International Conference on Scale-Space Theory in Computer Vision (Scale-Space'97), B. ter Haar Romeny, L. Florack, J. Koenderink, and M. Viergever, Eds., vol. 1252 of Lecture Notes in Computer Science, pp. 323-326, Springer, Utrecht, The Netherlands, July 1997.

[67] M. J. Black, G. Sapiro, D. H. Marimont, and D. Heeger, "Robust anisotropic diffusion," IEEE Transactions on Image Processing, vol. 7, no. 3, pp. 421-432, 1998.

[68] M. J. Black and G. Sapiro, "Edges as outliers: anisotropic smoothing using local image statistics," in Proceedings of the 2nd International Conference on Scale-Space Theories in Computer Vision (Scale-Space '99), vol. 1682 of Lecture Notes in Computer Science, pp. 259-270, Springer, Corfu, Greece, September 1999.

[69] V. Patanavijit and S. Jitapunkul, "A robust iterative multiframe super-resolution reconstruction using a Bayesian approach with lorentzian norm," in Proceedings of the 10th IEEE International Conference on Communication Systems (ICCS '06), pp. 1-5, Singapore, October 2006.

Patanavijit received the B.Eng. and M.Eng. degrees from the Department of Electrical Engineering at the Chulalongkorn University, Bangkok, Thailand, in 1994 and 1997 , respectively. He is currently pursuing the Doctoral degree (D.Eng.) in electrical engineering at Chulalongkorn University, Bangkok, Thailand. He is currently a full-time lecturer at Department of Computer Engineering, Faculty of Engineering,

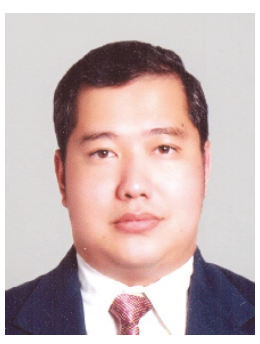
Assumption University. He works in the field of signal processing and multidimensional signal processing, specializing, in particular, on image/video reconstruction, super-resolution reconstruction (SRR), enhancement, fusion, denoising, inverse problems, motion estimation and registration.

Jitapunkul received the B.Eng. and M.Eng. degrees in electrical engineering in 1972 and 1974, respectively, from Chulalongkorn University, Thailand. In 1976 and 1978, he received the D.E.A. and Dr. Ing. degrees, respectively, in "signaux et systems spatiotemporels” from Aix-Marseille University, France. He was appointed as a lecturer in the Department of Electrical Engineering at Chulalongkorn University in 1972, an Assis-

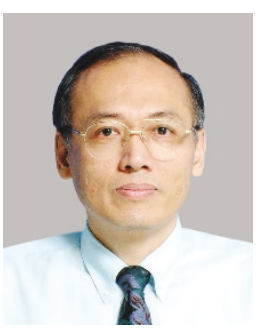
tant Professor in 1980, and an Associate Professor in 1983. In 1993, he was the founder of Digital Signal Processing Research Laboratory where he became the head of this laboratory from 1993 to 1997. From 1997 to 1999 and 1999 to 2003, he was appointed as the head of Communication Division and the head of the Department, respectively. He also held the position of Associate Dean for Information Technology, Faculty of Engineering from 1993 to 1995. His current research interests are in image and video processing, speech and character recognition, signal compression, DSP in telecommunication, software defined radio, smart antenna, and medical signal processing. 